\title{
RANDOM BIT MULTILEVEL ALGORITHMS FOR STOCHASTIC DIFFERENTIAL EQUATIONS
}

\author{
MICHAEL B. GILES, MARIO HEFTER, LUKAS MAYER, AND KLAUS RITTER
}

\begin{abstract}
We study the approximation of expectations $\mathrm{E}(f(X))$ for solutions $X$ of SDEs and functionals $f: C\left([0,1], \mathbb{R}^{r}\right) \rightarrow \mathbb{R}$ by means of restricted Monte Carlo algorithms that may only use random bits instead of random numbers. We consider the worst case setting for functionals $f$ from the Lipschitz class w.r.t. the supremum norm. We construct a random bit multilevel Euler algorithm and establish upper bounds for its error and cost. Furthermore, we derive matching lower bounds, up to a logarithmic factor, that are valid for all random bit Monte Carlo algorithms, and we show that, for the given quadrature problem, random bit Monte Carlo algorithms are at least almost as powerful as general randomized algorithms.
\end{abstract}

\section{INTRODUCTION}

We study the approximation of expectations $\mathrm{E}(f(X))$, where $X=(X(t))_{t \in[0,1]}$ is the $r$ dimensional solution of an autonomous SDE with deterministic initial value and Lipschitz continuous drift and diffusion coefficients, driven by a $d$-dimensional Brownian motion. Moreover, $f \in \operatorname{Lip}_{1}$, i.e.,

$$
f: C\left([0,1], \mathbb{R}^{r}\right) \rightarrow \mathbb{R}
$$

is Lipschitz continuous with respect to the supremum norm with Lipschitz constant at most one.

We consider randomized (Monte Carlo) algorithms that are only allowed to use random bits instead of random numbers. By assumption, all other operations (arithmetic operations, evaluations of elementary functions, and oracle calls to evaluate $f$ as well as the drift and diffusion coefficients of the SDE) are performed exactly. Algorithms of this type are called random bit Monte Carlo algorithms, and the approximation of expectations by algorithms of this type is called random bit quadrature. Due to the path-dependence of $f$ the approximation of $\mathrm{E}(f(X))$ is an infinite-dimensional quadrature problem.

In a worst case setting algorithms $A$ are compared according to their worst case error $\mathrm{e}\left(A, \operatorname{Lip}_{1}\right)$ and their worst case $\operatorname{cost} \operatorname{cost}\left(A, \operatorname{Lip}_{1}\right)$ on the class $\operatorname{Lip}_{1}$. The cost takes into account, in particular, the number of random bits used and the information cost, i.e., the cost for the evaluations of $f$. For the latter, we suppose that any $f \in \operatorname{Lip}_{1}$ can be evaluated at any piecewise linear function $x \in C\left([0,1], \mathbb{R}^{r}\right)$ with equidistant breakpoints $0,1 / 2^{\ell}, \ldots, 1$ at cost $2^{\ell}+1$ for any $\ell \in \mathbb{N}_{0}$. See Sections 2 , 5.1, and 5.3 for details.

The main contribution of this paper is the construction of a random bit multilevel Euler algorithm $A_{\varepsilon}^{\text {Bbit }}$ that is almost optimal. First of all, we have the following upper bound:

Date: January 16, 2019.

2010 Mathematics Subject Classification. 60H35, 60H10, 65D30, 65C05.

Key words and phrases. random bits, multilevel Monte Carlo algorithms, stochastic differential equations. 
There exists a constant $c>0$ such that

$$
\mathrm{e}\left(A_{\varepsilon}^{\mathrm{Bbit}}, \operatorname{Lip}_{1}\right) \leq c \cdot \varepsilon
$$

and

$$
\operatorname{cost}\left(A_{\varepsilon}^{\text {Bbit }}, \operatorname{Lip}_{1}\right) \leq c \cdot \varepsilon^{-2} \cdot\left(\ln \left(\varepsilon^{-1}\right)\right)^{3}
$$

for every $\varepsilon \in] 0,1 / 2[$, see Theorem 8 . This result coincides with the best known upper bound for general randomized algorithms, which are achieved by the classical multilevel Euler algorithm. See Giles [9] for a survey on multilevel algorithms.

An important ingredient for the construction of $A_{\varepsilon}^{\text {Bbit }}$ is Bakhvalov's trick: A small number of independent random variables, each uniformly distributed on $\left\{1, \ldots, 2^{q}\right\}$, yields a much larger number of pairwise independent random variables with the same uniform distribution. The number of random bits used by the algorithm $A_{\varepsilon}^{\text {Bbit }}$ is of the order $\varepsilon^{-2} \cdot\left(\ln \left(\varepsilon^{-1}\right)\right)^{5 / 2}$, see the proof of Theorem 8 , and it can be reduced further to $\varepsilon^{-2}$. $\left(\ln \left(\varepsilon^{-1}\right)\right)^{2} \cdot \ln \left(\ln \left(\varepsilon^{-1}\right)\right)$, as outlined in Remark 9. Since the upper bound in (1) is sharp, the number of random bits is asymptotically negligible compared to the overall cost of $A_{\varepsilon}^{\text {Bbit }}$.

Secondly, the algorithm $A_{\varepsilon}^{\text {Bbit }}$ is optimal, up to logarithmic factors. Under a slightly stronger smoothness assumption as well as a non-degeneracy assumption on the diffusion coefficient of the SDE, which in particular exclude pathological cases yielding a deterministic solution $X$, the following holds true: There exist constants $c, \varepsilon_{0}>0$ such that

$$
\operatorname{cost}\left(A, \operatorname{Lip}_{1}\right) \geq c \cdot \varepsilon^{-2}
$$

for every random bit Monte Carlo algorithm $A$ and for every $\left.\varepsilon \in] 0, \varepsilon_{0}\right]$ such that

$$
\mathrm{e}\left(A, \operatorname{Lip}_{1}\right) \leq \varepsilon
$$

Actually, there are two variants of this result, both of which hold for a much broader class of algorithms. In the first variant, the evaluation of $f$ is allowed at arbitrary points $x \in C\left([0,1], \mathbb{R}^{r}\right)$ at cost one, while the number of random bits is taken into account as before, see Theorem [15. In the second variant, which is due to Creutzig et al. [7, Thm. 11], roughly speaking, any kind of randomness is allowed for free, but the cost model with evaluations of $f$ only at piecewise linear functions is kept. We do not know whether random bits are as powerful as random numbers for the quadrature problem under investigation, but the upper and lower bounds imply that random bits are at least almost as powerful as random numbers.

This work is a continuation of Giles et al. [10]. Two approximation problems involving random bits have been considered there: Random bit quadrature, as in the present paper, and random bit approximation of probability measures, which is closely related to quantization. Furthermore, two classes of processes have been considered there: Gaussian processes and solution processes of SDEs. For three of the possible four combinations results have already been obtained in Giles et al. [10]. The combination of random bit quadrature for SDEs, which remained open, is addressed in the present paper.

This work is partially motivated by reconfigurable architectures like field programmable gate arrays (FPGAs). These devices allow users to choose the precision of each individual operation on a bit level and provide a generator for random bits. In the setting and analysis of the present paper we take into account the latter fact, while we ignore all finite precision issues for arithmetic operations. We refer to Brugger et al. [5], Omland et al. 
[19] for the construction and for extensive tests of a finite precision multilevel algorithm for FPGAs with applications in computational finance. For an error analysis of the Euler scheme for SDEs in a finite precision arithmetic we refer to Omland [18]. A complete analysis on a bit level for the arithmetic operations, the random number generator, and the oracle is not yet available.

In most of the papers on randomized algorithms for continuous problems, uniformly distributed random numbers from $[0,1]$ are assumed to be available. Random bit Monte Carlo algorithms are studied for the classical, finite-dimensional quadrature problem to approximate $\int_{[0,1]^{d}} f(x) \mathrm{d} x$ in, e.g., Gao et al. [8], Heinrich et al. [12], Novak [14, 15, 16], Traub and Woźniakowski [20], Ye and Hu [22]. See Novak and Pfeiffer [17] for a related approach to integral equations.

In Heinrich et al. [12], random bit quadrature with respect to the uniform distribution on $[0,1]^{d}$ and Sobolev and Hölder classes of functions $f:[0,1]^{d} \rightarrow \mathbb{R}$ are considered. It is shown that random bit Monte Carlo algorithms are as powerful as general randomized algorithms, and a very small number of random bits suffice to achieve asymptotic optimality. The proofs of these results are based on a reduction of the quadrature problem to a summation problem and on Bakhvalov's trick.

In contrast to, e.g., Heinrich et al. [12], we do not derive lower bounds that separately take into account the number of random bits and the information cost. Instead, we establish lower bounds in terms of the overall cost, which includes, in particular, the sum of both of these quantities. A general framework to study Monte Carlo algorithms that only have access to generators for an arbitrary, but fixed set of probability distributions has recently been introduced in Heinrich [11]. Within the latter framework the information cost and the cost associated to the calls of the available random number generators are naturally studied separately.

This paper is organized as follows. In Section 2 we formulate the computational problem. Section 3 is devoted to the definition and strong error analysis of a random bit Euler scheme. In Section 4 we present the construction of the random bit multilevel Euler algorithm with corresponding error and cost bounds. Lower bounds for random bit Monte Carlo methods are derived in Section 5. In Appendix A we present Bakhvalov's trick in a form that fits to our needs.

\section{The Computational Problem}

Let $r, d \in \mathbb{N}$. Henceforth we use $|\cdot|$ to denote the Euclidean norm, and we consider the corresponding supremum norm $\|\cdot\|$ on $C\left([0,1], \mathbb{R}^{r}\right)$.

We consider an autonomous system

$$
\mathrm{d} X(t)=a(X(t)) \mathrm{d} t+b(X(t)) \mathrm{d} W(t), \quad t \in[0,1]
$$

of SDEs with a deterministic initial value

$$
X(0)=x_{0} \in \mathbb{R}^{r}
$$

and a $d$-dimensional Brownian motion $W$, and with Lipschitz-continuous drift and diffusion coefficients $a: \mathbb{R}^{r} \rightarrow \mathbb{R}^{r}$ and $b: \mathbb{R}^{r} \rightarrow \mathbb{R}^{r \times d}$, respectively. We study the approximation of

$$
S(f)=\mathrm{E}(f(X))
$$


for functionals $f: C\left([0,1], \mathbb{R}^{r}\right) \rightarrow \mathbb{R}$ that are Lipschitz continuous with Lipschitz constant at most one, i.e.,

$$
|f(x)-f(y)| \leq\|x-y\|
$$

for all $x, y \in C\left([0,1], \mathbb{R}^{r}\right)$. The class of all such Lipschitz functionals $f$ is denoted by $\operatorname{Lip}_{1}$.

We employ the real-number model, i.e., we assume that algorithms can perform comparisons, arithmetic operations on real numbers, and the evaluation of elementary functions at unit cost. Furthermore, we assume that the drift coefficient $a$ and the diffusion coefficient $b$ can be evaluated at each point $x \in \mathbb{R}^{r}$ at unit cost. Moreover, we suppose that any functional $f \in \operatorname{Lip}_{1}$ can be evaluated at any piecewise linear function with breakpoints $k / 2^{\ell}$ for $k=0, \ldots, 2^{\ell}$ at cost $2^{\ell}+1$ for any $\ell \in \mathbb{N}_{0}$.

Monte Carlo algorithms have access to a random number generator at cost one per call. Here, we distinguish two cases, namely algorithms with access to random numbers from $[0,1]$, and algorithms with access to random bits only, which we refer to as random bit Monte Carlo algorithms.

The cost, $\operatorname{cost}(A, f)$, of applying the Monte Carlo algorithm $A$ to the functional $f$ is defined as the sum of the cost associated to every instruction that is carried out. Observe that under appropriate measurability assumptions $\operatorname{cost}(A, f)$ is a random quantity. We define the worst case cost of $A$ on the class $\operatorname{Lip}_{1}$ as

$$
\operatorname{cost}\left(A, \operatorname{Lip}_{1}\right)=\sup _{f \in \operatorname{Lip}_{1}} \mathrm{E}(\operatorname{cost}(A, f)) .
$$

Since the output $A(f)$ of a Monte Carlo algorithm $A$ applied to an input functional $f$ from $\mathrm{Lip}_{1}$ is a random quantity, too, its error is defined by

$$
\mathrm{e}(A, f)=\left(\mathrm{E}|S(f)-A(f)|^{2}\right)^{1 / 2}
$$

under appropriate measurability assumptions. Accordingly, the worst case error on the class $\operatorname{Lip}_{1}$ is defined by

$$
\mathrm{e}\left(A, \operatorname{Lip}_{1}\right)=\sup _{f \in \operatorname{Lip}_{1}} \mathrm{e}(A, f)
$$

See Section 5.1 for a rigorous definition of a more general cost model that only takes into account the information cost concerning $f$ and the number of random bits used.

\section{Euler Schemes}

The key ingredient for the construction and analysis of random bit multilevel algorithms, see Section 4, is a random bit Euler scheme and strong error bounds thereof, which will be presented in Sections 3.2 and 3.3 .

Let

$$
t_{k}=t_{k, m}=k / m
$$

where $m \in \mathbb{N}$ and $k=0, \ldots, m$. A corresponding Euler scheme is given by

$$
\begin{aligned}
& X_{m}\left(t_{0, m}\right)=x_{0} \\
& X_{m}\left(t_{k, m}\right)=X_{m}\left(t_{k-1, m}\right)+m^{-1} \cdot a\left(X_{m}\left(t_{k-1, m}\right)\right)+b\left(X_{m}\left(t_{k-1, m}\right)\right) \cdot V_{k, m}
\end{aligned}
$$


with suitable random vectors $V_{k, m}$ to be defined below. The multilevel approach relies on a coupling of $X_{m}$ with an even number $m \in \mathbb{N}$ of steps to an Euler scheme

$$
\begin{aligned}
\widetilde{X}_{m / 2}\left(t_{0, m / 2}\right) & =x_{0}, \\
\widetilde{X}_{m / 2}\left(t_{k, m / 2}\right)=\widetilde{X}_{m / 2}\left(t_{k-1, m / 2}\right) & +(m / 2)^{-1} \cdot a\left(\widetilde{X}_{m / 2}\left(t_{k-1, m / 2}\right)\right) \\
& +b\left(\widetilde{X}_{m / 2}\left(t_{k-1, m / 2}\right)\right) \cdot \widetilde{V}_{k, m / 2}
\end{aligned}
$$

with $m / 2$ steps. Suitable random vectors $\widetilde{V}_{k, m / 2}$ will be defined below.

In order to approximate $X$ at any point $t \in[0,1]$ we extend $X_{m}\left(t_{0}\right), \ldots, X_{m}\left(t_{m}\right)$ and $\widetilde{X}_{m / 2}\left(t_{0}\right), \ldots, \widetilde{X}_{m / 2}\left(t_{m / 2}\right)$ by linear interpolation onto the subintervals $] t_{k-1}, t_{k}[$.

3.1. The Classical Euler Scheme. In the vast majority of papers, $X_{m}$ is based on the Brownian increments

$$
V_{k, m}=W\left(t_{k, m}\right)-W\left(t_{k-1, m}\right)
$$

which are naturally coupled via

$$
\widetilde{V}_{k, m / 2}=V_{2 k, m}+V_{2 k-1, m}=W\left(t_{k, m / 2}\right)-W\left(t_{k-1, m / 2}\right) .
$$

Obviously, $\widetilde{V}_{k, m / 2}=V_{k, m / 2}$, which is convenient in the analysis of multilevel algorithms. For the corresponding classical Euler scheme we use the notation $X_{m}^{\mathrm{c}}$ and $\widetilde{X}_{m / 2}^{\mathrm{c}}$, and likewise we use $V_{k, m}^{\mathrm{c}}$ and $\widetilde{V}_{k, m / 2}^{\mathrm{c}}$ for the corresponding increments.

3.2. A Random Bit Euler Scheme. In the present paper we study an Euler scheme that only uses random bits instead of random numbers from $[0,1]$. This excludes the use of Brownian increments. At first we discuss the approximation of standard normal distributions based on random bits.

Let $\Phi$ denote the distribution function of $N(0,1)$ with inverse function $\Phi^{-1}$, and let $Y \sim N(0,1)$. We introduce the rounding function

$$
T^{(q)}:\left[0,1\left[\rightarrow D^{(q)}, \quad x \mapsto \frac{\left\lfloor 2^{q} x\right\rfloor}{2^{q}}+2^{-(q+1)},\right.\right.
$$

where $q \in \mathbb{N}$ and

$$
D^{(q)}=\left\{\sum_{i=1}^{q} b_{i} \cdot 2^{-i}+2^{-(q+1)}: b_{i} \in\{0,1\} \text { for } i=1, \ldots, q\right\} .
$$

Then

$$
Y^{(q)}=\Phi^{-1} \circ T^{(q)} \circ \Phi(Y)
$$

serves as a canonical approximation of $Y$.

Observe that $T^{(q)} \circ \Phi(Y)$ is uniformly distributed on $D^{(q)}$. Consequently, $q$ random bits suffice to simulate the distribution of $Y^{(q)}$. Further properties of $Y^{(q)}$ have been established in Giles et al. [10], see also Remark 10 below. For a standard normally distributed random vector $Y$ an approximation $Y^{(q)}$ is obtained by applying $\Phi^{-1} \circ T^{(q)} \circ \Phi$ to each of the components of $Y$ separately.

We use this approximation in a straightforward way, i.e., we study a random bit Euler scheme with

$$
V_{k, m}=m^{-1 / 2} \cdot\left(m^{1 / 2} \cdot\left(W\left(t_{k, m}\right)-W\left(t_{k-1, m}\right)\right)\right)^{(q)}
$$


A suitable coupling is easily achieved by

$$
\widetilde{V}_{k, m / 2}=V_{2 k, m}+V_{2 k-1, m},
$$

cf. (3). To indicate the dependence of this coupled Euler scheme on the bit number $q$ we use the notation $X_{m, q}^{\text {bit }}$ and $\widetilde{X}_{m / 2, q}^{\text {bit }}$, and likewise $V_{k, m, q}^{\text {bit }}$ and $\widetilde{V}_{k, m / 2, q}^{\text {bit }}$ for the approximations of the Brownian increments. Proper relations between the number $q$ of bits and the number $m$ of Euler steps will be presented in Section 4 .

The simulation of the joint distribution of $X_{m, q}^{\text {bit }}$ and $\widetilde{X}_{m / 2, q}^{\text {bit }}$ requires $d \cdot m \cdot q$ random bits. We stress that the distributions of $\widetilde{V}_{k, m / 2, q}^{\text {bit }}$ and $V_{k, m / 2, q}^{\text {bit }}$ do not coincide, and therefore

$$
\mathrm{E}\left(f\left(X_{m / 2}\right)\right) \neq \mathrm{E}\left(f\left(\widetilde{X}_{m / 2}\right)\right)
$$

in general. This introduces an additional bias term in the multilevel analysis, cf. Müller et al. [13, Thm. 6.1].

3.3. Strong Error Analysis. It is well known that there exists a constant $c>0$ such that

$$
\left(\mathrm{E}\left\|X-X_{m}^{\mathrm{c}}\right\|^{2}\right)^{1 / 2} \leq c \cdot m^{-1 / 2} \cdot(\ln (m+1))^{1 / 2}
$$

for every $m \in \mathbb{N}$. Thus we will provide an upper bound for the difference between the random bit Euler scheme $X_{m, q}^{\text {bit }}$ and the classical Euler scheme $X_{m}^{\mathrm{c}}$. The difference between $\widetilde{X}_{m / 2, q}^{\text {bit }}$ and $\widetilde{X}_{m / 2}^{\mathrm{c}}$ may be treated in the same way.

Remark 1. We gather some properties of the random vectors $V_{k, m}^{\mathrm{c}}$ and $V_{k, m}^{\mathrm{bit}}$ and of the scheme $X_{m, q}^{\mathrm{bit}}$.

(a) We have independence of $\left(V_{k, m, q}^{\mathrm{bit}}\right)_{k=1, \ldots, m}$.

(b) There exists a constant $c>0$ such that

$$
\left(\mathrm{E}\left|V_{k, m}^{\mathrm{c}}-V_{k, m, q}^{\mathrm{bit}}\right|^{2}\right)^{1 / 2} \leq c \cdot m^{-1 / 2} \cdot 2^{-q / 2} \cdot q^{-1 / 2}
$$

and

$$
\mathrm{E}\left(V_{k, m, q}^{\mathrm{bit}}\right)=0
$$

for all $m \in \mathbb{N}, k=1, \ldots, m$, and $q \in \mathbb{N}$. Furthermore, we have

$$
\sup _{m \in \mathbb{N}} \sup _{k=1, \ldots, m} \sup _{q \in \mathbb{N}}\left(m^{1 / 2} \cdot\left(\mathrm{E}\left|V_{k, m, q}^{\mathrm{bit}}\right|^{r}\right)^{1 / r}\right)<\infty
$$

for all $r \geq 1$. See Giles et al. [10, Thm. 1].

(c) We have E $\left\|X_{m, q}^{\text {bit }}\right\|^{2}<\infty$ for every $m \in \mathbb{N}$ and $q \in \mathbb{N}$.

In the sequel, we assume that the Lipschitz constants of the drift coefficient $a$ and of the diffusion coefficient $b$ are bounded by $\gamma$.

Lemma 2. There exists a constant $c>0$ such that for all $m, q \in \mathbb{N}$ we have

$$
\max _{k=0, \ldots, m}\left(\mathrm{E}\left|X_{m}^{\mathrm{c}}\left(t_{k}\right)-X_{m, q}^{\mathrm{bit}}\left(t_{k}\right)\right|^{2}\right)^{1 / 2} \leq c \cdot 2^{-q / 2} \cdot q^{-1 / 2}
$$


Proof. This proof follows the standard analysis for the classical Euler scheme.

For $k=0, \ldots, m-1$ we have

$$
X_{m}^{\mathrm{c}}\left(t_{k+1}\right)-X_{m, q}^{\mathrm{bit}}\left(t_{k+1}\right)=\xi+\zeta,
$$

where

$$
\xi=X_{m}^{\mathrm{c}}\left(t_{k}\right)-X_{m, q}^{\mathrm{bit}}\left(t_{k}\right)+m^{-1} \cdot\left(a\left(X_{m}^{\mathrm{c}}\left(t_{k}\right)\right)-a\left(X_{m, q}^{\mathrm{bit}}\left(t_{k}\right)\right)\right)
$$

and

$$
\zeta=b\left(X_{m}^{\mathrm{c}}\left(t_{k}\right)\right) \cdot V_{k+1, m}^{\mathrm{c}}-b\left(X_{m, q}^{\mathrm{bit}}\left(t_{k}\right)\right) \cdot V_{k+1, m, q}^{\mathrm{bit}} .
$$

For any pair of components $\xi_{i}$ and $\zeta_{i}$ of $\xi$ and $\zeta$, respectively, we have

$$
\mathrm{E}\left(\xi_{i} \cdot \zeta_{i}\right)=0
$$

due to properties (a)-(c) from Remark 1. It follows that

$$
\mathrm{E}\left|X_{m}^{\mathrm{c}}\left(t_{k+1}\right)-X_{m, q}^{\mathrm{bit}}\left(t_{k+1}\right)\right|^{2}=\mathrm{E}\left(|\xi|^{2}\right)+\mathrm{E}\left(|\zeta|^{2}\right) .
$$

The Lipschitz continuity of $a$ yields

$$
\left(\mathrm{E}|\xi|^{2}\right)^{1 / 2} \leq(1+\gamma / m) \cdot\left(\mathrm{E}\left|X_{m}^{\mathrm{c}}\left(t_{k}\right)-X_{m, q}^{\mathrm{bit}}\left(t_{k}\right)\right|^{2}\right)^{1 / 2} .
$$

Moreover,

$$
\begin{aligned}
\mathrm{E}\left(|\zeta|^{2}\right) \leq & 2 \cdot\left(\mathrm{E}\left|b\left(X_{m}^{\mathrm{c}}\left(t_{k}\right)\right) \cdot\left(V_{k+1, m}^{\mathrm{c}}-V_{k+1, m, q}^{\mathrm{bit}}\right)\right|^{2}\right. \\
& \left.+\mathrm{E}\left|\left(b\left(X_{m}^{\mathrm{c}}\left(t_{k}\right)\right)-b\left(X_{m, q}^{\mathrm{bit}}\left(t_{k}\right)\right)\right) \cdot V_{k+1, m, q}^{\mathrm{bit}}\right|^{2}\right) .
\end{aligned}
$$

Due to the independence of $\left(V_{k, m}^{\mathrm{c}}\right)_{k=1, \ldots, m}$ we have

$$
\mathrm{E}\left|b\left(X_{m}^{\mathrm{c}}\left(t_{k}\right)\right) \cdot\left(V_{k+1, m}^{\mathrm{c}}-V_{k+1, m, q}^{\mathrm{bit}}\right)\right|^{2} \leq \mathrm{E}\left|b\left(X_{m}^{\mathrm{c}}\left(t_{k}\right)\right)\right|^{2} \cdot \mathrm{E}\left|V_{k+1, m}^{\mathrm{c}}-V_{k+1, m, q}^{\mathrm{bit}}\right|^{2} .
$$

Using

$$
\mathrm{E}\left|b\left(X_{m}^{\mathrm{c}}\left(t_{k}\right)\right)\right|^{2} \leq 2 \cdot \gamma^{2} \cdot \mathrm{E}\left|X_{m}^{\mathrm{c}}\left(t_{k}\right)\right|^{2}+2 \cdot|b(0)|^{2}
$$

together with property (b) from Remark 1 we obtain a constant $c_{1}>0$ such that

$$
\mathrm{E}\left|b\left(X_{m}^{\mathrm{c}}\left(t_{k}\right)\right) \cdot\left(V_{k+1, m}^{\mathrm{c}}-V_{k+1, m, q}^{\mathrm{bit}}\right)\right|^{2} \leq c_{1} \cdot m^{-1} \cdot 2^{-q} \cdot q^{-1}
$$

for all $m, q \in \mathbb{N}$ and $k=0, \ldots, m-1$. Property (a) from Remark 1 also gives

$$
\mathrm{E}\left|\left(b\left(X_{m}^{\mathrm{c}}\left(t_{k}\right)\right)-b\left(X_{m, q}^{\mathrm{bit}}\left(t_{k}\right)\right)\right) \cdot V_{k+1, m, q}^{\mathrm{bit}}\right|^{2} \leq \mathrm{E}\left|b\left(X_{m}^{\mathrm{c}}\left(t_{k}\right)\right)-b\left(X_{m, q}^{\mathrm{bit}}\left(t_{k}\right)\right)\right|^{2} \cdot \mathrm{E}\left|V_{k+1, m, q}^{\mathrm{bit}}\right|^{2} .
$$

Moreover,

$$
\mathrm{E}\left|b\left(X_{m}^{\mathrm{c}}\left(t_{k}\right)\right)-b\left(X_{m, q}^{\mathrm{bit}}\left(t_{k}\right)\right)\right|^{2} \leq \gamma^{2} \cdot \mathrm{E}\left|X_{m}^{\mathrm{c}}\left(t_{k}\right)-X_{m, q}^{\mathrm{bit}}\left(t_{k}\right)\right|^{2},
$$

and property (b) from Remark 1 yields the existence of a constant $c_{2}>0$ such that

$$
\mathrm{E}\left|\left(b\left(X_{m}^{\mathrm{c}}\left(t_{k}\right)\right)-b\left(X_{m, q}^{\mathrm{bit}}\left(t_{k}\right)\right)\right) \cdot V_{k+1, m, q}^{\mathrm{bit}}\right|^{2} \leq c_{2} \cdot m^{-1} \cdot \mathrm{E}\left|X_{m}^{\mathrm{c}}\left(t_{k}\right)-X_{m, q}^{\mathrm{bit}}\left(t_{k}\right)\right|^{2}
$$

for all $m, q \in \mathbb{N}$ and $k=0, \ldots, m-1$. It follows that

$$
\mathrm{E}\left(|\zeta|^{2}\right) \leq c_{3} / m \cdot\left(2^{-q} \cdot q^{-1}+\mathrm{E}\left|X_{m}^{\mathrm{c}}\left(t_{k}\right)-X_{m, q}^{\mathrm{bit}}\left(t_{k}\right)\right|^{2}\right)
$$


with $c_{3}=2 \cdot \max \left(c_{1}, c_{2}\right)$. Combining (8) and (9) we get the existence of a constant $c>0$ such that

$$
\mathrm{E}\left|X_{m}^{\mathrm{c}}\left(t_{k+1}\right)-X_{m, q}^{\mathrm{bit}}\left(t_{k+1}\right)\right|^{2} \leq(1+c / m) \cdot \mathrm{E}\left|X_{m}^{\mathrm{c}}\left(t_{k}\right)-X_{m, q}^{\mathrm{bit}}\left(t_{k}\right)\right|^{2}+c / m \cdot 2^{-q} \cdot q^{-1}
$$

for all $m, q \in \mathbb{N}$ and $k=0, \ldots, m-1$.

A discrete Gronwall-inequality or a straightforward computation yields

$$
\begin{aligned}
& \mathrm{E}\left|X_{m}^{\mathrm{c}}\left(t_{k+1}\right)-X_{m, q}^{\mathrm{bit}}\left(t_{k+1}\right)\right|^{2} \\
& \quad \leq(1+c / m)^{k+1} \cdot \mathrm{E}\left|X_{m}^{\mathrm{c}}\left(t_{0}\right)-X_{m, q}^{\mathrm{bit}}\left(t_{0}\right)\right|^{2}+\sum_{j=0}^{k}(1+c / m)^{j} \cdot c / m \cdot 2^{-q} \cdot q^{-1} \\
& \quad \leq(1+c / m)^{m} \cdot c / m \cdot(k+1) \cdot 2^{-q} \cdot q^{-1}
\end{aligned}
$$

with $c$ according to (10), and hereby the statement for $X_{m}^{\mathrm{c}}-X_{m, q}^{\mathrm{bit}}$ follows.

Lemma 3. There exists a constant $c>0$ such that for all $m, q \in \mathbb{N}$ we have

$$
\left(\mathrm{E}\left\|X_{m}^{\mathrm{c}}-X_{m, q}^{\mathrm{bit}}\right\|^{2}\right)^{1 / 2} \leq c \cdot 2^{-q / 2} \cdot q^{-1 / 2}
$$

Proof. This proof uses standard martingale arguments to exchange the maximum with the expectation in the error bound of Lemma 2 .

It suffices to consider the error at the point $t_{k}$, since $X_{m}^{\mathrm{c}}$ and $X_{m, q}^{\mathrm{bit}}$ are piecewise linear with breakpoints $t_{k}$. For $k=1, \ldots, m$ we have

$$
\begin{aligned}
X_{m}^{\mathrm{c}}\left(t_{k}\right)-X_{m, q}^{\mathrm{bit}}\left(t_{k}\right)= & m^{-1} \cdot \sum_{\ell=1}^{k}\left(a\left(X_{m}^{\mathrm{c}}\left(t_{\ell-1}\right)\right)-a\left(X_{m, q}^{\mathrm{bit}}\left(t_{\ell-1}\right)\right)\right) \\
& +\sum_{\ell=1}^{k}\left(b\left(X_{m}^{\mathrm{c}}\left(t_{\ell-1}\right)\right) \cdot V_{\ell, m}^{\mathrm{c}}-b\left(X_{m, q}^{\mathrm{bit}}\left(t_{\ell-1}\right)\right) \cdot V_{\ell, m, q}^{\mathrm{bit}}\right),
\end{aligned}
$$

and therefore

$$
\mathrm{E} \max _{k=0, \ldots, m}\left|X_{m}^{\mathrm{c}}\left(t_{k}\right)-X_{m, q}^{\mathrm{bit}}\left(t_{k}\right)\right|^{2} \leq 2 \cdot \mathrm{E} \max _{k=1, \ldots, m}\left|Z_{k}\right|^{2}+2 \cdot \mathrm{E} \max _{k=1, \ldots, m}\left|R_{k}\right|^{2}
$$

with

$$
Z_{k}=m^{-1} \cdot \sum_{\ell=1}^{k}\left(a\left(X_{m}^{\mathrm{c}}\left(t_{\ell-1}\right)\right)-a\left(X_{m, q}^{\mathrm{bit}}\left(t_{\ell-1}\right)\right)\right)
$$

and

$$
R_{k}=\sum_{\ell=1}^{k}\left(b\left(X_{m}^{\mathrm{c}}\left(t_{\ell-1}\right)\right) \cdot V_{\ell, m}^{\mathrm{c}}-b\left(X_{m, q}^{\mathrm{bit}}\left(t_{\ell-1}\right)\right) \cdot V_{\ell, m, q}^{\mathrm{bit}}\right) .
$$

For the drift term we have

$$
\begin{aligned}
\mathrm{E} \max _{k=1, \ldots, m}\left|Z_{k}\right|^{2} & \leq \mathrm{E} \max _{k=1, \ldots, m} \frac{k}{m^{2}} \sum_{\ell=1}^{k}\left|a\left(X_{m}^{\mathrm{c}}\left(t_{\ell-1}\right)\right)-a\left(X_{m, q}^{\mathrm{bit}}\left(t_{\ell-1}\right)\right)\right|^{2} \\
& \leq \frac{\gamma^{2}}{m} \cdot \sum_{\ell=1}^{m} \mathrm{E}\left|X_{m}^{\mathrm{c}}\left(t_{\ell-1}\right)-X_{m, q}^{\mathrm{bit}}\left(t_{\ell-1}\right)\right|^{2} \\
& \leq \gamma^{2} \cdot \max _{k=0, \ldots, m} \mathrm{E}\left|X_{m}^{\mathrm{c}}\left(t_{k}\right)-X_{m, q}^{\mathrm{bit}}\left(t_{k}\right)\right|^{2}
\end{aligned}
$$


For the diffusion term, we note that $\left(R_{k}\right)_{k=1, \ldots, m}$ is a martingale due to properties (b) and (c) from Remark 1, Consequently, the Doob maximal inequality yields

$$
\mathrm{E} \max _{k=1, \ldots, m}\left|R_{k}\right|^{2} \leq 4 \cdot \mathrm{E}\left|R_{m}\right|^{2} .
$$

Use (11) and (12) to obtain

$$
\begin{aligned}
\mathrm{E}\left|R_{m}\right|^{2} & =\mathrm{E}\left|X_{m}^{\mathrm{c}}\left(t_{m}\right)-X_{m, q}^{\mathrm{bit}}\left(t_{m}\right)-Z_{m}\right|^{2} \\
& \leq 2 \cdot \mathrm{E}\left|X_{m}^{\mathrm{c}}\left(t_{m}\right)-X_{m, q}^{\mathrm{bit}}\left(t_{m}\right)\right|^{2}+2 \cdot \mathrm{E}\left|Z_{m}\right|^{2} \\
& \leq 2 \cdot\left(1+\gamma^{2}\right) \cdot \max _{k=0, \ldots, m} \mathrm{E}\left|X_{m}^{\mathrm{c}}\left(t_{k}\right)-X_{m, q}^{\mathrm{bit}}\left(t_{k}\right)\right|^{2} .
\end{aligned}
$$

We conclude that

$$
\mathrm{E} \max _{k=0, \ldots, m}\left|X_{m}^{\mathrm{c}}\left(t_{k}\right)-X_{m, q}^{\mathrm{bit}}\left(t_{k}\right)\right|^{2} \leq 18 \cdot\left(1+\gamma^{2}\right) \cdot \max _{k=0, \ldots, m} \mathrm{E}\left|X_{m}^{\mathrm{c}}\left(t_{k}\right)-X_{m, q}^{\mathrm{bit}}\left(t_{k}\right)\right|^{2} .
$$

Apply Lemma 2 to derive the statement for $X_{m}^{\mathrm{c}}-X_{m, q}^{\mathrm{bit}}$.

Remark 4. Let $\Delta$ denote the Wasserstein distance of order two on the space of all Borel probability measures on a separable Banach space. For any such measure $\mu$ the asymptotics of

$$
\operatorname{rbit}(\mu, p)=\inf \left\{\Delta(\mu, \nu): \nu \text { uniform distribution with support of size } 2^{p}\right\}, \quad p \in \mathbb{N},
$$

have been recently studied in Berger and Xu [3, 4], Chevallier [6], Giles et al. [10], Xu and Berger [21]. Combining Lemma 3 with (7) we get the existence of a constant $c>0$ such that

$$
\operatorname{rbit}(\mu, p) \leq c \cdot \min _{d \cdot m \cdot q \leq p}\left(m^{-1 / 2} \cdot(\ln (m+1))^{1 / 2}+2^{-q / 2} \cdot q^{-1 / 2}\right)
$$

for all $p \in \mathbb{N}$, where $\mu$ is the distribution of the solution $X$ on the Banach space $C\left([0,1], \mathbb{R}^{r}\right)$. It can be shown that the right hand side of $(13)$ is of the order $p^{-1 / 2} \cdot \ln (p)$. In the scalar case, i.e., $r=d=1$, and under a slightly stronger smoothness assumption on the coefficients $a, b$ as well as a non-degeneracy assumption on the diffusion coefficients $b$ of the SDE, which in particular exclude pathological cases yielding a deterministic solution, it has been shown in Giles et al. [10, Thm. 4] that $\operatorname{rbit}(\mu, p)$ is of the order $p^{-1 / 2}$ for the Banach space $L_{2}[0,1]$. Hence the upper bound obtained via (13) is sharp, up to logarithmic factors, but matching upper and lower bounds seem to be unknown in this case.

Following the proofs of Lemma 2 and Lemma 3, one may establish analogous results for the difference $\widetilde{X}_{m / 2}^{\mathrm{c}}-\widetilde{X}_{m / 2, q}^{\mathrm{bit}}$. We only formulate the analogue to Lemma 3 ,

Lemma 5. There exists a constant $c>0$ such that for every $q \in \mathbb{N}$ and every even $m \in \mathbb{N}$ we have

$$
\left(\mathrm{E}\left\|\widetilde{X}_{m / 2}^{\mathrm{c}}-\widetilde{X}_{m / 2, q}^{\mathrm{bit}}\right\|^{2}\right)^{1 / 2} \leq c \cdot 2^{-q / 2} \cdot q^{-1 / 2}
$$

Lemma 3 and Lemma [5, together with (7), will be used to control the variances and the bias of multilevel algorithms in the following section. Concerning the variances, a different approach is provided in Belomestny and Nagapetyan [2, Sec. 3.1]. 


\section{Multilevel Euler Algorithms}

At first we give a general description of a multilevel algorithm based on either of the two Euler schemes in Section [3. Let $L \in \mathbb{N}$ be the maximum level that is used by this algorithm. On every level $\ell=1, \ldots, L$ the algorithm involves a fine approximation $X_{2^{\ell}}$ and a coarse approximation $\widetilde{X}_{2^{\ell-1}}$ according to Section 3 . On level $\ell=0$ we only need the fine approximation $X_{2^{0}}$, but we set $\widetilde{X}_{2^{-1}}=0$ for notational convenience. Furthermore, let $N=\left(N_{0}, \ldots, N_{L}\right) \in \mathbb{N}^{L+1}$ be the vector of replication numbers on the levels $\ell=0, \ldots, L$.

To define the multilevel algorithm we consider a family

$$
\boldsymbol{X}_{L, N}=\left(\left(X_{2^{\ell}, i}, \widetilde{X}_{2^{\ell-1}, i}\right)\right)_{\ell=0, \ldots, L, i=1, \ldots, N_{\ell}}
$$

of random elements $\left(X_{2^{\ell}, i}, \widetilde{X}_{2^{\ell-1}, i}\right)$ such that

$$
\left(X_{2^{\ell}, i}, \widetilde{X}_{2^{\ell-1}, i}\right) \stackrel{\mathrm{d}}{=}\left(X_{2^{\ell}}, \widetilde{X}_{2^{\ell-1}}\right)
$$

for $\ell=0, \ldots, L$ and $i=1, \ldots, N_{\ell}$. The joint distribution of $\boldsymbol{X}_{L, N}$ will be specified later. The multilevel Euler algorithm finally reads as

$$
A_{L, N}(f)=\frac{1}{N_{0}} \cdot \sum_{i=1}^{N_{0}} f\left(X_{2^{0}, i}\right)+\sum_{\ell=1}^{L} \frac{1}{N_{\ell}} \cdot \sum_{i=1}^{N_{\ell}}\left(f\left(X_{2^{\ell}, i}\right)-f\left(\widetilde{X}_{2^{\ell-1}, i}\right)\right) .
$$

Subsequently we study three different variants of this algorithm. In the first two variants $\boldsymbol{X}_{L, N}$ is independent, i.e., the family $\boldsymbol{X}_{L, N}$ of random variables $\left(X_{2^{\ell}, i}, \widetilde{X}_{2^{\ell-1}, i}\right)$ is independent, which is a standard assumption for multilevel algorithms. In the first case we use the classical Euler scheme with normally distributed increments. In the other two cases we consider random bit Monte Carlo algorithms. In the second case we simply employ random bit approximations of the normally distributed increments. In the third case we apply Bakhvalov's trick in order to reduce the number of random bits; consequently, $\boldsymbol{X}_{L, N}$ is no longer independent.

4.1. The Classical Multilevel Algorithm. In this section $\boldsymbol{X}_{L, N}$ is assumed to be independent and

$$
\left(X_{2^{\ell}}, \widetilde{X}_{2^{\ell-1}}\right) \stackrel{\mathrm{d}}{=}\left(X_{2^{\ell}}^{\mathrm{c}}, \widetilde{X}_{2^{\ell-1}}^{\mathrm{c}}\right)
$$

for $\ell=0, \ldots, L$, where $\widetilde{X}_{2^{-1}}^{\mathrm{c}}=0$ for notational convenience. See Section 3.1. Hereby we get the classical multilevel Euler algorithm, which we denote by $A_{L, N}^{\mathrm{c}}$.

We estimate the cost of $A_{L, N}^{\mathrm{c}}$. For that purpose we consider the cost on level $\ell$. The cost for the arithmetic operations, the evaluations of $a, b$ and $f$ as well as $\Phi^{-1}$ on level $\ell$ is, up to a multiplicative constant, given by $N_{\ell} \cdot 2^{\ell}$. The number $r_{\ell}$ of calls to the random number generator on level $\ell$ is given by

$$
r_{\ell}=N_{\ell} \cdot 2^{\ell} \cdot d
$$

Consequently, there exists a constant $c>1$ such that for all $L$ and $N$ the multilevel Euler algorithm satisfies

$$
c^{-1} \cdot \sum_{\ell=0}^{L} 2^{\ell} \cdot N_{\ell} \leq \operatorname{cost}\left(A_{L, N}^{\mathrm{c}}, \operatorname{Lip}_{1}\right) \leq c \cdot \sum_{\ell=0}^{L} 2^{\ell} \cdot N_{\ell} .
$$

For $\varepsilon \in] 0,1 / 2[$ we consider the algorithm

$$
A_{\varepsilon}^{\mathrm{c}}=A_{L(\varepsilon), N(\varepsilon)}^{\mathrm{c}}
$$


with maximal level

$$
L=L(\varepsilon)=\left\lceil\log _{2}\left(\varepsilon^{-2}\right)+\log _{2}\left(\log _{2}\left(\varepsilon^{-2}\right)\right)\right\rceil
$$

and with replication numbers

$$
N_{\ell}=N_{\ell}(\varepsilon)=\left\lceil(L+1) \cdot 2^{-\ell} \cdot \max (\ell, 1) \cdot \varepsilon^{-2}\right\rceil
$$

for $\ell=0, \ldots, L$.

The following result is known, see, e.g., Creutzig et al. [7, Rem. 8]. For convenience of the reader we present a proof.

Theorem 6. There exists a constant $c>1$ such that the multilevel Euler algorithm $A_{\varepsilon}^{\mathrm{c}}$ satisfies

$$
\mathrm{e}\left(A_{\varepsilon}^{\mathrm{c}}, \operatorname{Lip}_{1}\right) \leq c \cdot \varepsilon
$$

and

$$
c^{-1} \cdot \varepsilon^{-2} \cdot\left(\ln \left(\varepsilon^{-1}\right)\right)^{3} \leq \operatorname{cost}\left(A_{\varepsilon}^{\mathrm{c}}, \operatorname{Lip}_{1}\right) \leq c \cdot \varepsilon^{-2} \cdot\left(\ln \left(\varepsilon^{-1}\right)\right)^{3}
$$

for every $\varepsilon \in] 0,1 / 2[$.

Proof. We show that both the squared bias and the variance of $A_{\varepsilon}^{\mathrm{c}}$ are bounded by $\varepsilon^{2}$, up to a constant. Use (7) to obtain a constant $c_{1}>0$ such that

$$
\mathrm{E}\left\|X-X_{2^{\ell}}^{\mathrm{c}}\right\|^{2} \leq c_{1} \cdot 2^{-\ell} \cdot \max (\ell, 1)
$$

for every $\ell \geq 0$ and $\mathrm{E}\left\|X_{2^{0}}\right\|^{2} \leq c_{1}$. Hence we get

$$
\left|S(f)-\mathrm{E}\left(A_{L, N}^{\mathrm{c}}(f)\right)\right|^{2}=\left|\mathrm{E}(f(X))-\mathrm{E}\left(f\left(X_{2^{L}}^{\mathrm{c}}\right)\right)\right|^{2} \leq c_{1} \cdot 2^{-L} \cdot L
$$

for all $L$ and $N$ and for every $f \in \operatorname{Lip}_{1}$. With $L=L(\varepsilon)$ this upper bound is of the order $\varepsilon^{2}$, as claimed. For the variance we get

$$
\operatorname{Var}\left(A_{L, N}^{\mathrm{c}}(f)\right) \leq 6 c_{1} \cdot \sum_{\ell=0}^{L} \frac{\max (\ell, 1)}{2^{\ell} \cdot N_{\ell}}
$$

for all $L$ and $N$ and for every $f \in \operatorname{Lip}_{1}$. With $N=N(\varepsilon)$ and $L=L(\varepsilon)$ this upper bound is of the order $\varepsilon^{2}$, too.

To derive the cost bounds for $A_{\varepsilon}^{\mathrm{c}}$ it remains to observe that there exists a constant $c_{2}>1$ such that

$$
c_{2}^{-1} \cdot \varepsilon^{-2} \cdot\left(\ln \left(\varepsilon^{-1}\right)\right)^{3} \leq \sum_{\ell=0}^{L(\varepsilon)} N_{\ell}(\varepsilon) \cdot 2^{\ell} \leq c_{2} \cdot \varepsilon^{-2} \cdot\left(\ln \left(\varepsilon^{-1}\right)\right)^{3}
$$

for all $\varepsilon$.

4.2. A Random Bit Multilevel Algorithm. In this section we consider a multilevel Euler algorithm that is based on random bits. As before, we assume that $\boldsymbol{X}_{L, N}$ is independent, but now we take

$$
\left(X_{2^{\ell}}, \widetilde{X}_{2^{\ell-1}}\right) \stackrel{\mathrm{d}}{=}\left(X_{2^{\ell}, q}^{\mathrm{bit}}, \widetilde{X}_{2^{\ell-1}, q}^{\mathrm{bit}}\right)
$$

for $\ell=0, \ldots, L$ and $q \in \mathbb{N}$, where $\widetilde{X}_{2^{-1}, q}^{\text {bit }}=0$ for notational convenience. See Section 3.2 . The resulting algorithm is denoted by $A_{L, N, q}^{\text {bit }}$.

A different construction of a random bit multilevel algorithm, which is based on the coupling (5) and which ensures that

$$
\widetilde{X}_{2^{\ell-1}} \stackrel{\mathrm{d}}{=} X_{2^{\ell-1}}
$$


is satisfied, is studied in Belomestny and Nagapetyan [2]. Recall that (15) is violated in our construction, except in trivial cases, see (6).

The cost of $A_{L, N, q}^{\text {bit }}$ can be bounded as in Section 4.1. In fact, the number $r_{\ell}$ of calls to the random number generator on level $\ell$ is given by

$$
r_{\ell}=N_{\ell} \cdot 2^{\ell} \cdot d \cdot q
$$

Furthermore, there exists a constant $c>1$ such that for all $L, N$, and $q$ the multilevel Euler algorithm satisfies

$$
c^{-1} \cdot q \cdot \sum_{\ell=0}^{L} 2^{\ell} \cdot N_{\ell} \leq \operatorname{cost}\left(A_{L, N, q}^{\mathrm{bit}}, \operatorname{Lip}_{1}\right) \leq c \cdot q \cdot \sum_{\ell=0}^{L} 2^{\ell} \cdot N_{\ell}
$$

For $\varepsilon \in] 0,1 / 2[$ we choose the bit number

$$
q(\varepsilon)=L(\varepsilon)
$$

and $L(\varepsilon)$ as well as $N(\varepsilon)$ as in Section 4.1 to obtain the algorithm

$$
A_{\varepsilon}^{\mathrm{bit}}=A_{L(\varepsilon), N(\varepsilon), q(\varepsilon)}^{\mathrm{bit}} .
$$

Theorem 7. There exists a constant $c>1$ such that the random bit multilevel Euler algorithm $A_{\varepsilon}^{\text {bit }}$ satisfies

$$
\mathrm{e}\left(A_{\varepsilon}^{\mathrm{bit}}, \operatorname{Lip}_{1}\right) \leq c \cdot \varepsilon
$$

and

$$
c^{-1} \cdot \varepsilon^{-2} \cdot\left(\ln \left(\varepsilon^{-1}\right)\right)^{4} \leq \operatorname{cost}\left(A_{\varepsilon}^{\mathrm{bit}}, \operatorname{Lip}_{1}\right) \leq c \cdot \varepsilon^{-2} \cdot\left(\ln \left(\varepsilon^{-1}\right)\right)^{4}
$$

for every $\varepsilon \in] 0,1 / 2[$.

Proof. At first we establish the error bound. Due to Theorem 6] it suffices to show the existence of a constant $c>0$ such that

$$
\sup _{f \in \operatorname{Lip}_{1}}\left(\mathrm{E}\left|A_{\varepsilon}^{\mathrm{c}}(f)-A_{\varepsilon}^{\mathrm{bit}}(f)\right|^{2}\right)^{1 / 2} \leq c \cdot \varepsilon
$$

for every $\varepsilon \in] 0,1 / 2\left[\right.$. For $f \in \operatorname{Lip}_{1}$ we have

$$
\left(\mathrm{E}\left|A_{L, N}^{\mathrm{c}}(f)-A_{L, N, q}^{\mathrm{bit}}(f)\right|^{2}\right)^{1 / 2} \leq \sum_{\ell=0}^{L}\left(\mathrm{E}\left\|X_{2^{\ell}}^{\mathrm{c}}-X_{2^{\ell}, q}^{\mathrm{bit}}\right\|^{2}\right)^{1 / 2}+\sum_{\ell=1}^{L}\left(\mathrm{E}\left\|\widetilde{X}_{2^{\ell-1}}^{\mathrm{c}}-\widetilde{X}_{2^{\ell-1}, q}^{\mathrm{bit}}\right\|^{2}\right)^{1 / 2}
$$

Apply Lemma 3 as well as Lemma 5 to obtain the existence of a constant $c_{1}>0$ such that

$$
\left(\mathrm{E}\left|A_{L, N}^{\mathrm{c}}(f)-A_{L, N, q}^{\mathrm{bit}}(f)\right|^{2}\right)^{1 / 2} \leq c_{1} \cdot L \cdot 2^{-q / 2} \cdot q^{-1 / 2}
$$

for all $L, q$, and $N$ and every $f \in \operatorname{Lip}_{1}$. With $q=L=L(\varepsilon)$ this upper bound is of the order $\varepsilon$.

The cost bounds follow immediately from (14). 
4.3. A Random Bit Multilevel Algorithm Based on Bakhvalov's Trick. In this section we consider a variant of the random bit multilevel algorithm $A_{L, N, q}^{\text {bit }}$ from Section [4.2, which is based on Bakhvalov's trick, see Bakhvalov [1] and also Heinrich et al. [12] and the references therein. In our case this trick yields $n^{2}$ pairwise independent random variables, each of which is uniformly distributed on $D^{(q)}$, from $2 n$ independent random variables, each of which is uniformly distributed on $D^{(q)}$, see Lemma[17] in Appen$\operatorname{dix}$ A. Of course, the same is true in the $d$-dimensional situation, i.e., with $D^{(q)}$ replaced by $\left(D^{(q)}\right)^{d}$.

We construct a random bit multilevel Euler algorithm $A_{L, N, q}^{\mathrm{Bbit}}$ that achieves the following properties at a reduced number of random bits compared to $A_{L, N, q}^{\mathrm{bit}}$ :

(i) The family $\boldsymbol{X}_{L, N}$ is pairwise independent.

(ii) For every $\ell=0, \ldots, L$ we have

$$
\left(X_{2^{\ell}}, \widetilde{X}_{2^{\ell-1}}\right) \stackrel{\mathrm{d}}{=}\left(X_{2^{\ell}, q}^{\mathrm{bit}}, \widetilde{X}_{2^{\ell-1}, q}^{\mathrm{bit}}\right)
$$

as in Section 4.2 ,

These properties already ensure that the expectations and the variances of $A_{L, N, q}^{\mathrm{bit}}(f)$ and $A_{L, N, q}^{\text {Bbit }}(f)$ coincide for every $f \in \operatorname{Lip}_{1}$, so that $\mathrm{e}\left(A_{L, N, q}^{\mathrm{bit}}, f\right)=\mathrm{e}\left(A_{L, N, q}^{\mathrm{Bbit}}, f\right)$. In particular,

$$
\mathrm{e}\left(A_{L, N, q}^{\mathrm{bit}}, \operatorname{Lip}_{1}\right)=\mathrm{e}\left(A_{L, N, q}^{\mathrm{Bbit}}, \operatorname{Lip}_{1}\right)
$$

We describe the construction of $A_{L, N, q}^{\mathrm{Bbit}}$ or, equivalently, the distribution of $\boldsymbol{X}_{L, N}$ in detail. Let

$$
n_{\ell}=\left\lceil N_{\ell}^{1 / 2}\right\rceil
$$

for $\ell=0, \ldots, L$. Consider an independent family

$$
\boldsymbol{G}=\left(G_{k, 2^{\ell}, j}\right)_{k=1, \ldots, 2^{\ell}, \ell=0, \ldots, L, j=1, \ldots, 2 n_{\ell}}
$$

of random vectors $G_{k, 2^{\ell}, j}$ that are uniformly distributed on $\left(D^{(q)}\right)^{d}$. For $x \in \mathbb{R}$ we denote by $x \bmod 1$ the fractional part of $x$, i.e., the real number $y \in[0,1[$ that satisfies $x-y \in \mathbb{Z}$. For $k=1, \ldots, 2^{\ell}, \ell=0, \ldots, L$, and $j_{1}, j_{2}=1, \ldots, n_{\ell}$ define

$$
U_{k, 2^{\ell},\left(j_{1}-1\right) n_{\ell}+j_{2}}=G_{k, 2^{\ell}, j_{1}}+G_{k, 2^{\ell}, j_{2}+n_{\ell}}+2^{-(q+1)} \cdot \mathbf{1} \bmod 1
$$

where the fractional part is taken in each component separately and $\mathbf{1} \in \mathbb{R}^{d}$ denotes the vector with all components equal to 1 . Let

$$
\boldsymbol{U}_{\ell}=\left(U_{k, 2^{\ell}, i}\right)_{k=1, \ldots, 2^{\ell}, i=1, \ldots, N_{\ell}}
$$

for $\ell=0, \ldots, L$. Obviously, the following holds true:

(I) The family $\left(\boldsymbol{U}_{\ell}\right)_{\ell=0, \ldots, L}$ is independent.

(II) For all $\ell=0, \ldots, L$ and $i=1, \ldots, N_{\ell}$ the family $\left(U_{k, 2^{\ell}, i}\right)_{k=1, \ldots, 2^{\ell}}$ is independent. Let

$$
\boldsymbol{U}_{\ell, i}=\left(U_{k, 2^{\ell}, i}\right)_{k=1, \ldots, 2^{\ell}}
$$

for $\ell=0, \ldots, L$ and $i=1, \ldots, N_{\ell}$. Lemma 17 from Appendix A yields:

(III) For every $\ell=0, \ldots, L$ the family $\left(\boldsymbol{U}_{\ell, i}\right)_{i=1, \ldots, N_{\ell}}$ is pairwise independent.

(IV) For all $k=1, \ldots, 2^{\ell}, \ell=0, \ldots, L$, and $i=1, \ldots, N_{\ell}$ the random vector $U_{k, 2^{\ell}, i}$ is uniformly distributed on $\left(D^{(q)}\right)^{d}$. 
For $k=1, \ldots, 2^{\ell}, \ell=0, \ldots, L$, and $i=1, \ldots, N_{\ell}$ let

$$
V_{k, 2^{\ell}, i}=2^{-\ell / 2} \cdot \Phi^{-1}\left(U_{k, 2^{\ell}, i}\right),
$$

where the function $\Phi^{-1}$ is applied to each of the components of the $d$-dimensional random vector $U_{k, 2^{\ell}, i}$ separately, cf. (4), and for $k=1, \ldots, 2^{\ell-1}, \ell=1, \ldots, L$, and $i=1, \ldots, N_{\ell}$ let

$$
\widetilde{V}_{k, 2^{\ell-1}, i}=V_{2 k, 2^{\ell}, i}+V_{2 k-1,2^{\ell}, i}
$$

cf. (5). Finally, for $\ell=0, \ldots, L$ and $i=1, \ldots, N_{\ell}$ the random element $X_{2^{\ell}, i}$ is given by the Euler scheme using the increments $\left(V_{k, 2^{\ell}, i}\right)_{k=1, \ldots, 2^{\ell}}$, for $\ell=1, \ldots, L$ and $i=$ $1, \ldots, N_{\ell}$ the random element $\widetilde{X}_{2^{\ell-1}, i}$ is given by the Euler scheme using the increments $\left(\widetilde{V}_{k, 2^{\ell-1}, i}\right)_{k=1, \ldots, 2^{\ell-1}}$, and for $\ell=0$ and $i=1, \ldots, N_{\ell}$ we set $\widetilde{X}_{2^{\ell-1}, i}=0$.

Observe that (I) and (III) imply (i). Furthermore, (II) and (IV) imply

$$
\left(V_{k, 2^{\ell}, i}\right)_{k=1, \ldots, 2^{\ell}} \stackrel{\mathrm{d}}{=}\left(V_{k, 2^{\ell}, q}^{\mathrm{bit}}\right)_{k=1, \ldots, 2^{\ell}}
$$

for $\ell=0, \ldots, L$ and $i=1, \ldots, N_{\ell}$, so that we also have (ii).

The cost of $A_{L, N, q}^{\mathrm{Bbit}}$ can be bounded as in Sections 4.1 and 4.2. The number of calls to the random number generator is the number of random bits needed to simulate the distribution of $\boldsymbol{G}$. Therefore it is given by $\sum_{\ell=0}^{L} r_{\ell}$ with

$$
r_{\ell}=2 n_{\ell} \cdot 2^{\ell} \cdot q \cdot d \text {. }
$$

We add that $r_{\ell}$ is the number of calls to the random number generator on level $\ell$. Furthermore, there exists a constant $c>1$ such that for all $L, N$, and $q$ the multilevel Euler algorithm satisfies

$$
c^{-1} \cdot \sum_{\ell=0}^{L}\left(2^{\ell} \cdot N_{\ell}+n_{\ell} \cdot 2^{\ell} \cdot q\right) \leq \operatorname{cost}\left(A_{L, N, q}^{\mathrm{Bbit}}, \operatorname{Lip}_{1}\right) \leq c \cdot \sum_{\ell=0}^{L}\left(2^{\ell} \cdot N_{\ell}+n_{\ell} \cdot 2^{\ell} \cdot q\right) .
$$

For $\varepsilon \in] 0,1 / 2[$ we consider the algorithm

$$
A_{\varepsilon}^{\mathrm{Bbit}}=A_{L(\varepsilon), N(\varepsilon), q(\varepsilon)}^{\mathrm{Bbit}}
$$

with $L(\varepsilon)$ and $N(\varepsilon)$ as in Section 4.1 and $q(\varepsilon)$ as in Section 4.2 .

Theorem 8. There exists a constant $c>1$ such that the random bit multilevel Euler algorithm $A_{\varepsilon}^{\text {Bbit }}$ satisfies

$$
\mathrm{e}\left(A_{\varepsilon}^{\mathrm{Bbit}}, \operatorname{Lip}_{1}\right) \leq c \cdot \varepsilon
$$

and

$$
c^{-1} \cdot \varepsilon^{-2} \cdot\left(\ln \left(\varepsilon^{-1}\right)\right)^{3} \leq \operatorname{cost}\left(A_{\varepsilon}^{\mathrm{Bbit}}, \operatorname{Lip}_{1}\right) \leq c \cdot \varepsilon^{-2} \cdot\left(\ln \left(\varepsilon^{-1}\right)\right)^{3}
$$

for every $\varepsilon \in] 0,1 / 2[$.

Proof. The error bound follows directly from (16) and Theorem 7 .

The cost bounds follow directly from (14) and the following observation. Let $n_{\ell}(\varepsilon)=$ $\left\lceil N_{\ell}^{1 / 2}(\varepsilon)\right]$. Then there exists a constant $c>1$ such that

$$
c^{-1} \cdot \varepsilon^{-2} \cdot\left(\ln \left(\varepsilon^{-1}\right)\right)^{3 / 2} \leq \sum_{\ell=0}^{L(\varepsilon)} n_{\ell}(\varepsilon) \cdot 2^{\ell} \leq c \cdot \varepsilon^{-2} \cdot\left(\ln \left(\varepsilon^{-1}\right)\right)^{3 / 2}
$$

for all $\varepsilon$. 
Up to a multiplicative constant the error and cost bounds for the algorithm $A_{\varepsilon}^{\mathrm{Bbit}}$, which uses only random bits, and the classical multilevel algorithm $A_{\varepsilon}^{\mathrm{c}}$, which uses random numbers from $[0,1]$, coincide. A proper comparison, based on lower bounds, of the corresponding classes of randomized algorithms is given in Section 5.3.

Remark 9. We sketch an algorithm that uses asymptotically fewer random bits than the algorithm $A_{\varepsilon}^{\text {Bbit }}$, but still has the desired distributional properties (i) and (ii). However, the overall cost will not be improved. The idea is to use the following variant of Lemma 17. Consider an independent family $\left(G_{i, j}\right)_{i=1,2, j=1, \ldots, n}$ of random variables that are uniformly distributed on $D^{(q)}$. Then the family $\left(G_{i_{1}, 1}+G_{i_{2}, 2}+\cdots+G_{i_{n}, n}+2^{-(q+1)} \cdot(n-1)\right.$ $\bmod 1)_{i_{1}=1,2, \ldots, i_{n}=1,2}$ is pairwise independent with each random variable being uniformly distributed on $D^{(q)}$. Proceeding similar to the construction of $A_{L, N, q}^{\text {Bbit }}$, we obtain an algorithm that only needs $d \cdot \sum_{\ell=0}^{L} 2^{\ell} \cdot q \cdot 2 \hat{n}_{\ell}$ random bits, where

$$
\hat{n}_{\ell}=\left\lceil\log _{2} N_{\ell}\right\rceil
$$

if $N_{\ell} \geq 2$ and $\hat{n}_{\ell}=1 / 2$ if $N_{\ell}=1$. With the choice of the parameters $L, N, q$ as above this number of bits is of the order $\varepsilon^{-2} \cdot\left(\ln \left(\varepsilon^{-1}\right)\right)^{2} \cdot \ln \left(\ln \left(\varepsilon^{-1}\right)\right)$. See Appendix B for the upper bound, and observe that the number of bits needed on the highest level is already of this order.

Recall that the number of random bits needed for the algorithm $A_{\varepsilon}^{\text {Bbit }}$ is of the order $\varepsilon^{-2} \cdot\left(\ln \left(\varepsilon^{-1}\right)\right)^{5 / 2}$, see the proof of Theorem 8 , and the number of random bits needed for one path on the finest level is given by $d \cdot q \cdot 2^{L}$, which is of the order $\varepsilon^{-2} \cdot\left(\ln \left(\varepsilon^{-1}\right)\right)^{2}$.

\section{Lower Bounds for Random Bit Monte Carlo Algorithms}

We derive a general lower bound for random bit Monte Carlo algorithms, which is applied to the quadrature problem for SDEs. At first, we modify a basic setting from information-based complexity in order to formally introduce the class of all random bit Monte Carlo algorithms, cf. Heinrich [11].

5.1. Random Bit Monte Carlo Algorithms. Consider a non-empty class $F$ of realvalued functions on a set $\mathfrak{X} \neq \emptyset$ and a mapping

$$
S: F \rightarrow \mathbb{R},
$$

which is to be approximated. By assumption, a random bit Monte Carlo algorithm has access to the functions $f \in F$ via an oracle (subroutine) that provides function values $f(x)$ for points $x \in \mathfrak{X}$ and to an ideal generator for random bits. This generator is modelled by the probability space $(\Omega, \mathfrak{A}, P)$, where

$$
\Omega=\{0,1\}^{\mathbb{N}}
$$

and where $\mathfrak{A}$ and $P$ denote the product $\sigma$-algebra and the product measure of the power set of $\{0,1\}$ and the uniform distribution on $\{0,1\}$, respectively. The cost per evaluation of $f \in F$ is modelled by a mapping

$$
\mathfrak{c}: \mathfrak{X} \rightarrow \mathbb{N} \cup\{\infty\},
$$

see Creutzig et al. [7, Sec. 2].

For any function $f \in F$, its sequential evaluation, which may be interlaced with calls to the random bit generator, is formally defined by a sequence of mappings

$$
\psi_{1}:\{0,1\} \rightarrow \mathfrak{X}
$$


and

$$
\psi_{\ell}:\{0,1\}^{\ell} \times \mathbb{R}^{\ell-1} \rightarrow \mathfrak{X}
$$

with $\ell \geq 2$. For every $f \in F$, the first step consists of a call to the random bit generator, which yields a bit $\omega_{1} \in\{0,1\}$, followed by the evaluation of $f$ at $\psi_{1}\left(\omega_{1}\right) \in \mathfrak{X}$. After $n$ steps $n$ bits $\omega_{1}, \ldots, \omega_{n} \in\{0,1\}$ have been obtained, and the function values

$$
y_{1}=f\left(\psi_{1}\left(\omega_{1}\right)\right)
$$

and

$$
y_{\ell}=f\left(\psi_{\ell}\left(\omega_{1}, \ldots, \omega_{\ell}, y_{1}, \ldots, y_{\ell-1}\right)\right)
$$

with $\ell=2, \ldots, n$ are known. Trivially, $\omega \mapsto\left(y_{1}, \ldots, y_{n}\right)$ yields a measurable mapping from $\Omega$ to $\mathbb{R}^{n}$ for all $f \in F$ and $n \in \mathbb{N}$. A decision to stop or to further evaluate $f$ is made after each step. This is formally described by a sequence of mappings

$$
\tau_{\ell}:\{0,1\}^{\ell} \times \mathbb{R}^{\ell} \rightarrow\{0,1\}
$$

with $\ell \geq 1$, and the total number $n(\omega, f)$ of evaluations of $f$ is given by

$$
n(\omega, f)=\min \left\{\ell \in \mathbb{N}: \tau_{\ell}\left(\omega_{1}, \ldots, \omega_{\ell}, y_{1}, \ldots, y_{\ell}\right)=1\right\} \in \mathbb{N} \cup\{\infty\}
$$

for $\omega \in \Omega$. Trivially, $n(\cdot, f)$ is a measurable mapping from $\Omega$ to $\mathbb{N} \cup\{\infty\}$. We assume that

$$
\forall f \in F: P(\{n(\cdot, f)<\infty\})=1 .
$$

Finally, a sequence of mappings

$$
\phi_{\ell}:\{0,1\}^{\ell} \times \mathbb{R}^{\ell} \rightarrow \mathbb{R}
$$

with $\ell \geq 1$ yields the approximation

$$
\widehat{S}(\omega, f)=\phi_{n(\omega, f)}\left(\omega_{1}, \ldots, \omega_{n(\omega, f)}, y_{1}, \ldots, y_{n(\omega, f)}\right)
$$

to $S(f)$ for all $\omega \in \Omega$ and $f \in F$ with $n(\omega, f)<\infty$. Otherwise, i.e., if $n(\omega, f)=\infty$, we put $\widehat{S}(\omega, f)=0$. Trivially, $\widehat{S}(\cdot, f)$ is a measurable mapping for every $f \in F$.

The tuple $\widehat{\mathcal{S}}=\left(\left(\psi_{\ell}\right)_{\ell},\left(\tau_{\ell}\right)_{\ell},\left(\phi_{\ell}\right)_{\ell}\right)$ will be considered as a random bit Monte Carlo algorithm, with algorithm being understood in a broad sense, cf. Section 2, and the class of all such algorithms is denoted by $\mathbb{S}^{\text {bit }}$. For $\widehat{\mathcal{S}} \in \mathbb{S}^{\text {bit }}$ the corresponding mapping $\widehat{S}: \Omega \times F \rightarrow \mathbb{R}$ will be called the input-output mapping induced by $\widehat{\mathcal{S}}$.

For $\ell \in \mathbb{N}$ let $\pi_{\ell}: \Omega \rightarrow\{0,1\}^{\ell}$ be given by

$$
\pi_{\ell}(\omega)=\left(\omega_{1}, \ldots, \omega_{\ell}\right) .
$$

The information cost and the error for applying the algorithm $\widehat{\mathcal{S}} \in \mathbb{S}^{\text {bit }}$ to $f \in F$ are defined by

$$
\operatorname{cost}_{\mathfrak{c}}(\widehat{\mathcal{S}}, f)=\mathrm{E}\left(\sum_{\ell=1}^{n(\cdot, f)} \mathfrak{c}\left(\psi_{\ell}\left(\pi_{\ell}(\cdot), y_{1}, \ldots, y_{\ell-1}\right)\right)\right)
$$

and

$$
\mathrm{e}(\widehat{\mathcal{S}}, f)=\left(\mathrm{E}|S(f)-\widehat{S}(\cdot, f)|^{2}\right)^{1 / 2}
$$

respectively. Obviously, $\operatorname{cost}_{\mathfrak{c}}(\widehat{\mathcal{S}}, f)$ is also an upper bound for the expected number $\mathrm{E}(n(\cdot, f))$ of random bits that are used by $\widehat{\mathcal{S}}$, when applied to $f$. The worst case information cost and the worst case error of $\widehat{\mathcal{S}}$ are defined by

$$
\operatorname{cost}_{\mathfrak{c}}(\widehat{\mathcal{S}})=\sup _{f \in F} \operatorname{cost}_{\mathfrak{c}}(\widehat{\mathcal{S}}, f)
$$


and

$$
\mathrm{e}(\widehat{\mathcal{S}})=\sup _{f \in F} \mathrm{e}(\widehat{\mathcal{S}}, f)
$$

respectively. The $\varepsilon$-complexity for the approximation of $S$ by means of random bit Monte Carlo algorithms is defined by

$$
\operatorname{comp}_{\mathfrak{c}}^{\text {bit }}(\varepsilon)=\inf \left\{\operatorname{cost}_{\mathfrak{c}}(\widehat{\mathcal{S}}): \widehat{\mathcal{S}} \in \mathbb{S}^{\text {bit }}, \mathrm{e}(\widehat{\mathcal{S}}) \leq \varepsilon\right\}
$$

for $\varepsilon>0$.

If for every $\ell \geq 1$ none of the mappings $\psi_{\ell}, \tau_{\ell}$, and $\phi_{\ell}$ depends on $\omega_{1}, \ldots, \omega_{\ell}$, then $\widehat{\mathcal{S}}$ is called a deterministic algorithm, and the corresponding proper subclass of $\mathbb{S}^{\text {bit }}$ is denoted by $\mathbb{S}^{\text {det }}$. For $\widehat{\mathcal{S}} \in \mathbb{S}^{\text {det }}$ the expectations may be dropped in the definitions of the worst case information cost and error, and the $\varepsilon$-complexity for the approximation of $S$ by means of deterministic algorithms is defined by

$$
\operatorname{comp}_{\mathfrak{c}}^{\operatorname{det}}(\varepsilon)=\inf \left\{\operatorname{cost}_{\mathfrak{c}}(\widehat{\mathcal{S}}): \widehat{\mathcal{S}} \in \mathbb{S}^{\operatorname{det}}, \mathrm{e}(\widehat{\mathcal{S}}) \leq \varepsilon\right\}
$$

for $\varepsilon>0$.

Monte Carlo algorithms from the class $\mathbb{S}^{\text {bit }}$ may only use random bits. Let us drop this constraint and consider randomized algorithms in general. A randomized algorithm is defined by any probability space $(\Omega, \mathfrak{A}, P)$ and a mapping $\widehat{\mathcal{S}}: \Omega \rightarrow \mathbb{S}^{\text {det }}$, which induces an input-output mapping $\widehat{S}: \Omega \times F \rightarrow \mathbb{R}$. Roughly speaking, $(\Omega, \mathfrak{A}, P)$ is the computational probability space, which may carry any kind of random number generator. If for a fixed $\omega \in \Omega$ all random numbers that may potentially be used in the computation are fixed in advance, then the randomized algorithm is turned into the deterministic algorithm $\widehat{\mathcal{S}}(\omega)$ with input-output mapping $\widehat{S}(\omega, \cdot)$. Under the appropriate measurability assumptions, the worst case error and the worst case information cost of a randomized algorithm $\widehat{\mathcal{S}}$ are defined analogously to (18) and (19). The class of all randomized algorithms that satisfy the measurability assumptions is denoted by $\mathbb{S}^{\text {ran }}$, and

$$
\operatorname{comp}_{\mathfrak{c}}^{\text {ran }}(\varepsilon)=\inf \left\{\operatorname{cost}_{\mathfrak{c}}(\widehat{\mathcal{S}}): \widehat{\mathcal{S}} \in \mathbb{S}^{\mathrm{ran}}, \mathrm{e}(\widehat{\mathcal{S}}) \leq \varepsilon\right\}
$$

is the $\varepsilon$-complexity for the approximation of $S$ by means of randomized algorithms. We refer to, e.g., Creutzig et al. [7, Sec. 2.3] for further details. Obviously,

$$
\operatorname{comp}_{\mathfrak{c}}^{\text {ran }}(\varepsilon) \leq \operatorname{comp}_{\mathfrak{c}}^{\text {bit }}(\varepsilon) \leq \operatorname{comp}_{\mathfrak{c}}^{\operatorname{det}}(\varepsilon)
$$

for every $\varepsilon>0$.

Remark 10. Every algorithm $\widehat{\mathcal{S}} \in \mathbb{S}^{\text {bit }}$ is asking for exactly one new random bit prior to a new function evaluation. In a refined model, this number of random bits could vary; observe that the latter is actually the case for the random bit multilevel algorithms that have been studied in Sections 4.2 and 4.3. On the other hand, cost one should be charged explicitly for every call of the random bit generator in a refined model. Formally an algorithm in this refined model may be turned into an algorithm $\widehat{\mathcal{S}} \in \mathbb{S}^{\text {bit }}$ by additional calls of the random bit generator and additional evaluations of $f$ at a fixed element $y \in \mathfrak{X}$ with no impact on the further computation. Observe that the expected cost for applying the original algorithm in the refined model to $f \in F$ is bounded from above by $\mathfrak{c}(y) \cdot \operatorname{cost}_{\mathfrak{c}}(\widehat{\mathcal{S}}, f)$.

We stress that even this refined model disregards further details of the real-number model, cf. Section 2. However, for the purpose of establishing lower bounds with unspecified multiplicative constants, it suffices to study algorithms $\widehat{\mathcal{S}} \in \mathbb{S}^{\text {bit }}$ and $\operatorname{cost}_{\mathfrak{c}}(\widehat{\mathcal{S}}, f)$, 
i.e., the information cost, in the non-trivial case that there exists an element $y \in \mathfrak{X}$ with $c(y)<\infty$.

The refined model is formally introduced in Heinrich [11]. In this model upper and lower bounds that separately take into account the number of random bits and the information cost may be established in a natural way. Furthermore, this framework allows to consider Monte Carlo algorithms that only have access to generators for an arbitrary, but fixed set $\mathcal{Q}$ of probability distributions. Random bit Monte Carlo algorithms, as studied in the present paper, correspond to the case $\mathcal{Q}=\{Q\}$ with $Q$ denoting the uniform distribution on $\{0,1\}$, while $\mathcal{Q}=\{Q\}$ with $Q$ denoting the uniform distribution on $[0,1]$ is most often considered for quadrature problems.

5.2. A General Lower Bound. Let $\widehat{\mathcal{S}}=\left(\left(\psi_{\ell}\right)_{\ell},\left(\tau_{\ell}\right)_{\ell},\left(\phi_{\ell}\right)_{\ell}\right) \in \mathbb{S}^{\text {bit }}$, and suppose that there exist $k, c \in \mathbb{N}$ with the following properties: For all $\omega \in \Omega, f \in F$, and $y_{1}, \ldots, y_{k-1} \in$ $\mathbb{R}$ we have $n(\omega, f)=k$ and

$$
\sum_{\ell=1}^{k} \mathfrak{c}\left(\psi_{\ell}\left(\omega_{1}, \ldots, \omega_{\ell}, y_{1}, \ldots, y_{\ell-1}\right)\right)=c
$$

i.e., $k$ random bits are used in any case and the information cost is deterministic and does not depend on $f$. Then $\widehat{\mathcal{S}}$ can be emulated by $2^{k}$ deterministic algorithms, each with information cost $c$ for every $f$, plus a random choice among the outputs of the deterministic algorithms. This observation can be generalized to any $\widehat{\mathcal{S}} \in \mathbb{S}^{\text {bit }}$, and it leads to a lower bound for $\operatorname{comp}_{\mathfrak{c}}^{\text {bit }}(\varepsilon)$ in terms of $\operatorname{comp}_{\mathfrak{c}}^{\text {det }}(\varepsilon)$. Under the particular assumptions mentioned above this lower bound has already been established in Heinrich et al. [12, Prop. 1].

Lemma 11. For every algorithm $\widehat{\mathcal{S}} \in \mathbb{S}^{\text {bit }}$ with $\operatorname{cost}_{\mathfrak{c}}(\widehat{\mathcal{S}})<\infty$ there exists an algorithm $\widehat{\mathcal{S}}^{*} \in \mathbb{S}^{\operatorname{det}}$ such that

$$
\operatorname{cost}_{\mathfrak{c}}\left(\widehat{\mathcal{S}}^{*}\right) \leq 2 \cdot\left(\operatorname{cost}_{\mathfrak{c}}(\widehat{\mathcal{S}})+1\right) \cdot 4^{\operatorname{cost}_{\mathfrak{c}}(\widehat{\mathcal{S}})+1}
$$

and

$$
\mathrm{e}\left(\widehat{\mathcal{S}}^{*}\right) \leq 2 \cdot \mathrm{e}(\widehat{\mathcal{S}})
$$

Proof. Let $\widehat{\mathcal{S}}=\left(\left(\psi_{\ell}\right)_{\ell},\left(\tau_{\ell}\right)_{\ell},\left(\phi_{\ell}\right)_{\ell}\right) \in \mathbb{S}^{\text {bit }}$ with input-output mapping $\widehat{S}$. We may assume that $\mathrm{e}(\widehat{\mathcal{S}})<\infty$, in addition to $\operatorname{cost}_{\mathfrak{c}}(\widehat{\mathcal{S}})<\infty$. Hence $\widehat{S}(\cdot, f)$ is integrable for every $f \in F$. Moreover, let $n(\cdot, \cdot)$ be the corresponding total number of evaluations. Put

$$
k=\left\lceil\operatorname{cost}_{\mathfrak{c}}(\widehat{\mathcal{S}})\right\rceil \in \mathbb{N} .
$$

For $f \in F$ we define

$$
A_{f}=\left\{\omega \in \Omega: \sum_{\ell=1}^{n(\omega, f)} \mathfrak{c}\left(\psi_{\ell}\left(\pi_{\ell}(\omega), y_{1}, \ldots, y_{\ell-1}\right)\right) \leq 2 k\right\}
$$

and

$$
\widehat{S}^{*}(f)=\mathrm{E}\left(\widehat{S}(\cdot, f) \mid A_{f}\right) .
$$

Since $P\left(A_{f}\right) \geq 1 / 2$, we get the error bound

$$
\left|S(f)-\widehat{S}^{*}(f)\right|=\left|S(f)-\mathrm{E}\left(\widehat{S}(\cdot, f) \mid A_{f}\right)\right| \leq \mathrm{E}\left(|S(f)-\widehat{S}(\cdot, f)| \mid A_{f}\right) \leq 2 \cdot \mathrm{e}(\widehat{\mathcal{S}}, f)
$$

for the conditional expectation $\widehat{S}^{*}(f)$. 
It remains to show that $\widehat{S}^{*}$ is the input-output mapping of an algorithm $\widehat{\mathcal{S}}^{*} \in \mathbb{S}^{\text {det }}$ with a cost bound as claimed. For $\ell \in \mathbb{N}$ let $\widetilde{\pi}_{\ell}:\{0,1\}^{\ell} \rightarrow \Omega$ be given by

$$
\tilde{\pi}_{\ell}(\omega)=(\omega, 0,0, \ldots) \text {. }
$$

Since $\mathfrak{c} \geq 1$, we have $n(\omega, f) \leq 2 k$ for $\omega \in A_{f}$. Hereby it follows that

$$
1_{A_{f}}=1_{A_{f}} \circ \tilde{\pi}_{2 k} \circ \pi_{2 k}
$$

and

$$
\widehat{S}(\cdot, f) \cdot 1_{A_{f}}=\widehat{S}(\cdot, f) \circ \widetilde{\pi}_{2 k} \circ \pi_{2 k} \cdot 1_{A_{f}}=\left(\widehat{S}(\cdot, f) \cdot 1_{A_{f}}\right) \circ \widetilde{\pi}_{2 k} \circ \pi_{2 k} .
$$

Furthermore, $\pi_{2 k}$ is uniformly distributed on $\{0,1\}^{2 k}$. Hence we get

$$
\widehat{S}^{*}(f)=\frac{1}{\left|B_{f}\right|} \cdot \sum_{\omega \in\{0,1\}^{2 k}} \widehat{S}\left(\widetilde{\pi}_{2 k}(\omega), f\right) \cdot 1_{B_{f}}(\omega),
$$

where

$$
B_{f}=\left\{\omega \in\{0,1\}^{2 k}: \tilde{\pi}_{2 k}(\omega) \in A_{f}\right\} .
$$

For every $\omega \in\{0,1\}^{2 k}$ and every $f \in F$ the information cost of simultaneously computing $1_{B_{f}}(\omega)$ and $\widehat{S}\left(\widetilde{\pi}_{2 k}(\omega), f\right) \cdot 1_{B_{f}}(\omega)$ is bounded by $2 k$. We conclude that there exists an algorithm $\widehat{\mathcal{S}}^{*} \in \mathbb{S}^{\text {det }}$ with input-output mapping $\widehat{S}^{*}$ and $\operatorname{cost}_{\mathfrak{c}}\left(\widehat{\mathcal{S}}^{*}\right) \leq 2 k \cdot 4^{k}$.

Theorem 12. There exist universal constants $c_{1}, c_{2}>0$ such that

$$
c_{1} \cdot \ln \left(\operatorname{comp}_{\mathfrak{c}}^{\operatorname{det}}(2 \varepsilon)\right)-c_{2} \leq \operatorname{comp}_{\mathfrak{c}}^{\text {bit }}(\varepsilon)
$$

for every $\varepsilon>0$ with $\operatorname{comp}_{\mathfrak{c}}^{\operatorname{det}}(2 \varepsilon)<\infty$. Furthermore, $\operatorname{comp}_{\mathfrak{c}}^{\text {bit }}(\varepsilon)=\infty$ if $\operatorname{comp}_{\mathfrak{c}}^{\operatorname{det}}(2 \varepsilon)=\infty$.

Proof. Let $\widehat{\mathcal{S}} \in \mathbb{S}^{\text {bit }}$ with $\mathrm{e}(\widehat{\mathcal{S}}) \leq \varepsilon$ and $\operatorname{cost}_{\mathfrak{c}}(\widehat{\mathcal{S}})<\infty$. Choose $\widehat{\mathcal{S}}^{*} \in \mathbb{S}^{\text {det }}$ according to Lemma 11, and put $z=\operatorname{cost}_{\mathfrak{c}}(\widehat{\mathcal{S}})+1$. We obtain

$$
\operatorname{comp}_{\mathfrak{c}}^{\operatorname{det}}(2 \varepsilon) \leq \operatorname{cost}_{\mathfrak{c}}\left(\widehat{\mathcal{S}}^{*}\right) \leq 2 z \cdot 4^{z} \leq 2 \cdot \exp ((1+\ln (4)) \cdot z)
$$

which yields the lower bound for $\operatorname{cost}_{\mathfrak{c}}(\widehat{\mathcal{S}})$ as claimed.

In many cases the lower bound from Theorem 12 is far from sharp, see, e.g., Heinrich et al. 12] for finite-dimensional quadrature problems. The lower bound is useful, however, for the one-dimensional case studied in Example 14, where we do not have any regularity, as well as for the quadrature problem for SDEs.

Remark 13. Suppose that $\mathfrak{X}$ is a Banach space, $F=\operatorname{Lip}_{1}$, and $S$ is defined by integration with respect to a Borel probability measure $\mu$ on $\mathfrak{X}$. It is well known that $\operatorname{comp}_{\mathfrak{c}}^{\text {det }}$ is determined by the quantization numbers of $\mu$ of order one, if $\mathfrak{c}=1$, see, e.g., Creutzig et al. [7]. This relation is exploited in the proof of Theorem 15 below.

We present a computational problem that is trivial when random numbers may be used, but unsolvable if only random bits are available.

Example 14 . Let $\mathfrak{X}=[0,1]$, let $F$ denote the class of all functions $f:[0,1] \rightarrow \mathbb{R}$ that are constant $\lambda$-a.e., and let

$$
S(f)=\int_{[0,1]} f \mathrm{~d} \lambda
$$


for $f \in F$. Consider $\mathfrak{c}=1$, which is most natural in the present setting, and let $\varepsilon>0$. Obviously $\operatorname{comp}_{\mathfrak{c}}^{\operatorname{det}}(\varepsilon)=\infty$, so that Theorem 12 yields

$$
\operatorname{comp}_{\mathfrak{c}}^{\text {bit }}(\varepsilon)=\infty \text {. }
$$

On the other hand, $S(f)=f(\omega)$ for $\lambda$-a.e. $\omega \in[0,1]$, and therefore

$$
\operatorname{comp}_{\mathfrak{c}}^{\text {ran }}(\varepsilon)=1 \text {. }
$$

The same phenomenon is present for Monte Carlo algorithms that only have access to any fixed set $\mathcal{Q}$ of probability distributions: For any such $\mathcal{Q}$ there exists a computational problem that is trivial for the class $\mathbb{S}^{\mathrm{ran}}$, but is unsolvable by means of Monte Carlo algorithms with generators only for the distributions from $\mathcal{Q}$. See Heinrich [11, Thm. 3.1].

5.3. A Lower Bound for Quadrature of SDEs. We return to the quadrature problem for SDEs, see Section 2, Hence we have

$$
\mathfrak{X}=C\left([0,1], \mathbb{R}^{r}\right)
$$

and

$$
F=\operatorname{Lip}_{1}
$$

and $S$ is given by (2). The function $\mathfrak{c}$ is given as follows. For $\ell \in \mathbb{N}_{0}$ let $\mathfrak{X}_{\ell}$ denote the set of all piecewise linear functions $x \in \mathfrak{X}$ with breakpoints $k / 2^{\ell}$ for $k=0, \ldots, 2^{\ell}$, so that the spaces $\mathfrak{X}_{\ell}$ form an increasing sequence of finite-dimensional subspaces of $\mathfrak{X}$, whose union is dense in $\mathfrak{X}$. Obviously, $\operatorname{dim}\left(\mathfrak{X}_{\ell}\right)=2^{\ell}+1$, and $\mathfrak{c}$ is defined by

$$
\mathfrak{c}(x)=\inf \left\{\operatorname{dim}\left(\mathfrak{X}_{\ell}\right): x \in \mathfrak{X}_{\ell}\right\}
$$

for $x \in \mathfrak{X}$. Compared to Sections 2 4, we impose slightly stronger smoothness assumption as well as a non-degeneracy assumption on the diffusion coefficient $b$ of the SDE, which in particular exclude pathological cases yielding a deterministic solution of the SDE.

Theorem 15. Assume that $r=d$ and that

- $b: \mathbb{R}^{r} \rightarrow \mathbb{R}^{r \times r}$ has bounded first and second order partial derivatives and is of class $C^{\infty}$ in some neighborhood of $x_{0}$,

- $\operatorname{det} b\left(x_{0}\right) \neq 0$,

in addition to the Lipschitz continuity of $a: \mathbb{R}^{r} \rightarrow \mathbb{R}^{r}$. Then there exist constants $c, \varepsilon_{0}>0$ such that

$$
\operatorname{comp}_{\mathfrak{c}}^{\text {bit }}(\varepsilon) \geq c \cdot \varepsilon^{-2}
$$

for every $\left.\varepsilon \in] 0, \varepsilon_{0}\right]$.

Proof. According to Creutzig et al. [7, Thm. 1, Prop. 3] there exist constants $c, \varepsilon_{0}>0$ such that $\operatorname{comp}_{1}^{\operatorname{det}}(\varepsilon) \geq \exp \left(c \cdot \varepsilon^{-2}\right)$ for every $\left.\left.\varepsilon \in\right] 0, \varepsilon_{0}\right]$. Apply Theorem 12.

We combine Theorems 8 and 15.

Corollary 16. Under the assumptions from Theorem 15 the following holds true: There exist constants $c_{1}, c_{2}, \varepsilon_{0}>0$ such that

$$
c_{1} \cdot \varepsilon^{-2} \leq \operatorname{comp}_{\mathfrak{c}}^{\text {bit }}(\varepsilon) \leq c_{2} \cdot \varepsilon^{-2} \cdot\left(\ln \left(\varepsilon^{-1}\right)\right)^{3}
$$

for every $\left.\varepsilon \in] 0, \varepsilon_{0}\right]$, and the random bit multilevel Euler algorithm $A_{\varepsilon}^{\mathrm{Bbit}}$ that achieves the upper bound is almost optimal. 
We stress that the lower bounds from Theorem [15] and from Corollary [16] hold even for $\mathfrak{c}=1$, i.e., in the most generous cost model, where every $f \in \operatorname{Lip}_{1}$ may be evaluated at any point $x \in C\left([0,1], \mathbb{R}^{r}\right)$ at unit cost. The key to prove the lower bound is the number of random bits together with the number of function evaluations being used by any algorithm $\widehat{\mathcal{S}} \in \mathbb{S}^{\text {bit }}$.

Due to Creutzig et al. [7, Thm. 11] we have the following lower bound for the class $\mathbb{S}^{\text {ran }}$ under the assumptions from Theorem 15: There exist constants $c_{1}, \varepsilon_{0}>0$ such that

$$
\operatorname{comp}_{\mathfrak{c}}^{\text {ran }}(\varepsilon) \geq c_{1} \cdot \varepsilon^{-2}
$$

for every $\left.\varepsilon \in] 0, \varepsilon_{0}\right]$. Obviously the latter estimate together with (20) yields a second proof of Theorem 15. We stress that this lower bound is no longer valid for $\mathfrak{c}=1$, i.e., it does not suffice to only consider the number of function evaluations being used by any algorithm $\widehat{\mathcal{S}} \in \mathbb{S}^{\mathrm{ran}}$. The lower bound is valid, however, for every $\mathfrak{c}$ according to (21) based on any increasing sequence of finite-dimensional subspaces $\mathfrak{X}_{\ell} \subset \mathfrak{X}$.

While the asymptotic behavior of $\operatorname{comp}_{\mathfrak{c}}^{\text {bit }}(\varepsilon)$ and $\operatorname{comp}_{\mathfrak{c}}^{\text {ran }}(\varepsilon)$ for $\varepsilon \rightarrow 0$ is not known exactly, their ratio is bounded by a multiple of $\left(\ln \left(\varepsilon^{-1}\right)\right)^{3}$. In particular, we do not know whether random bits are as powerful as random numbers for the quadrature problem under investigation, but random bits are at least almost as powerful as random numbers. Note that $\operatorname{comp}_{\mathfrak{c}}^{\text {bit }}(\varepsilon)$ is dramatically smaller than $\operatorname{comp}_{1}^{\operatorname{det}}(\varepsilon)$.

\section{Appendix A. BakhValov's Trick}

Lemma 17. Let $q \in \mathbb{N}$ and $n \in \mathbb{N}$. Consider an independent family $\left(G_{j}\right)_{j=1, \ldots, 2 n}$ of random variables that are uniformly distributed on $D^{(q)}$. Then the family $\left(G_{j_{1}}+G_{j_{2}+n}+2^{-(q+1)}\right.$ $\bmod 1)_{j_{1}, j_{2}=1, \ldots, n}$ is pairwise independent with each random variable being uniformly distributed on $D^{(q)}$.

Proof. Let $G$ be uniformly distributed on $D^{(q)}$. Inductively, we get

$$
\forall z \in 2^{-q} \cdot \mathbb{N}: z+G \bmod 1 \text { is uniformly distributed on } D^{(q)} .
$$

Furthermore,

$$
G+2^{-(q+1)} \in 2^{-q} \cdot \mathbb{N}
$$

Let $j_{1}, j_{2} \in\{1, \ldots, n\}$. Conditioned on $G_{j_{1}}$ the random variable $G_{j_{2}+n}$ is uniformly distributed on $D^{(q)}$. Combining this with (22) and (23) shows that conditioned on $G_{j_{1}}$ the random variable $G_{j_{1}}+G_{j_{2}+n}+2^{-(q+1)}$ mod 1 is uniformly distributed on $D^{(q)}$. Hence the random variable $G_{j_{1}}+G_{j_{2}+n}+2^{-(q+1)} \bmod 1$ is uniformly distributed on $D^{(q)}$.

Let $i_{1}, i_{2} \in\{1, \ldots, n\}$ such that $\left(i_{1}, i_{2}\right) \neq\left(j_{1}, j_{2}\right)$. Next we show that $G_{j_{1}}+G_{j_{2}+n}+$ $2^{-(q+1)} \bmod 1$ and $G_{i_{1}}+G_{i_{2}+n}+2^{-(q+1)} \bmod 1$ are independent. The case $i_{1} \neq j_{1}$ and $i_{2} \neq j_{2}$ is trivial. Thus without loss of generality we may assume $i_{1}=j_{1}$ and $i_{2} \neq j_{2}$. Conditioned on $G_{j_{1}}$ the random variables $G_{i_{2}+n}$ and $G_{j_{2}+n}$ are independent and each uniformly distributed on $D^{(q)}$. Combining this with (22) and (23) shows that conditioned on $G_{j_{1}}$ the random variables $G_{j_{1}}+G_{j_{2}+n}+2^{-(q+1)} \bmod 1$ and $G_{i_{1}}+G_{i_{2}+n}+2^{-(q+1)} \bmod 1$ are independent and each uniformly distributed on $D^{(q)}$. Hence we get the independence of the random variables $G_{j_{1}}+G_{j_{2}+n}+2^{-(q+1)} \bmod 1$ and $G_{i_{1}}+G_{i_{2}+n}+2^{-(q+1)} \bmod 1$. 


\section{Appendix B. An Estimate used in Remark 9}

Observe that

$$
\sum_{\ell=1}^{L} 2^{\ell} \cdot(L-\ell)=2^{L+1}-2(L+1) \leq 2^{L+1} .
$$

Hence there exist $\left.\varepsilon_{0} \in\right] 0,1 / 2\left[\right.$ and $c_{1}, c_{2}, c_{3}>0$ such that for every $\left.\varepsilon \in\right] 0, \varepsilon_{0}[$ we have

$$
\begin{aligned}
\sum_{\ell=0}^{L(\varepsilon)} 2^{\ell} \cdot 2 \hat{n}_{\ell}(\varepsilon) & \leq c_{1} \cdot \sum_{\ell=1}^{L(\varepsilon)} 2^{\ell} \cdot \log _{2}\left(L(\varepsilon) \cdot 2^{-\ell} \cdot \ell \cdot \varepsilon^{-2}\right) \\
& \leq c_{1} \cdot \sum_{\ell=1}^{L(\varepsilon)} 2^{\ell} \cdot\left(L(\varepsilon)-\ell+\log _{2}\left(L(\varepsilon)^{2}\right)+\log _{2}\left(\varepsilon^{-2}\right)-L(\varepsilon)\right) \\
& \leq c_{1} \cdot \sum_{\ell=1}^{L(\varepsilon)} 2^{\ell} \cdot\left(L(\varepsilon)-\ell+c_{2} \cdot \ln \left(\ln \left(\varepsilon^{-1}\right)\right)\right) \\
& \leq c_{1} \cdot\left(2^{L(\varepsilon)+1}+c_{2} \cdot \ln \left(\ln \left(\varepsilon^{-1}\right)\right) \cdot 2^{L(\varepsilon)+1}\right) \\
& \leq c_{3} \cdot \varepsilon^{-2} \cdot \ln \left(\varepsilon^{-1}\right) \cdot \ln \left(\ln \left(\varepsilon^{-1}\right)\right) .
\end{aligned}
$$

\section{ACKNOWLEDGMENT}

The authors are grateful to Steffen Omland for many valuable discussions and contributions at an early stage of this project. We thank Erich Novak for pointing out Example 14 to us, and Stefan Heinrich and Holger Stroot for valuable discussions and comments.

Mike Giles was partially supported by the UK Engineering and Physical Science Research Council (EPSRC) through the ICONIC Programme Grant, EP/P020720/1. Lukas Mayer was supported by the Deutsche Forschungsgemeinschaft (DFG) within the RTG 1932 'Stochastic Models for Innovations in the Engineering Sciences'.

\section{REFERENCES}

[1] N. S. Bakhvalov. Optimal convergence bounds for quadrature processes and integration methods of Monte Carlo type for classes of functions. Ž. Vyčisl. Mat. i Mat. Fiz., 4(4, suppl.):5-63, 1964.

[2] D. Belomestny and T. Nagapetyan. Multilevel path simulation for weak approximation schemes with application to Lévy-driven SDEs. Bernoulli, 23:927-950, 2017.

[3] A. Berger and C. Xu. The asymptotics of purely atomic one-dimensional Levy approximations. arXiv e-prints, Sept. 2018.

[4] A. Berger and C. Xu. Best finite approximations of Benford's law. J. Theoret. Probab., 2018. doi: 10.1007/s10959-018-0827-z.

[5] C. Brugger, C. De Schryver, N. Wehn, S. Omland, M. Hefter, K. Ritter, A. Kostiuk, and R. Korn. Mixed precision multilevel Monte Carlo on hybrid computing systems. In 2014 IEEE Conference on Computational Intelligence for Financial Engineering Economics (CIFEr), pages 215-222, March 2014.

[6] J. Chevallier. Uniform decomposition of probability measures: quantization, clustering and rate of convergence. J. Appl. Probab., 2018. In press.

[7] J. Creutzig, S. Dereich, T. Müller-Gronbach, and K. Ritter. Infinite-dimensional quadrature and approximation of distributions. Found. Comput. Math., 9(4):391429, 2009. 
[8] W. Gao, P. Ye, and H. Wang. Optimal error bound of restricted Monte Carlo integration on anisotropic Sobolev classes. Progr. Natur. Sci. (English Ed.), 16(6):588-593, 2006.

[9] M. B. Giles. Multilevel Monte Carlo methods. Acta Numer., 24:259-328, 2015.

[10] M. B. Giles, M. Hefter, L. Mayer, and K. Ritter. Random bit quadrature and approximation of distributions on Hilbert spaces. Found. Comput. Math., 2018. doi: 10.1007/s10208-018-9382-3.

[11] S. Heinrich. On the power of restricted Monte Carlo algorithms. Preprint, 2018.

[12] S. Heinrich, E. Novak, and H. Pfeiffer. How many random bits do we need for Monte Carlo integration? In Monte Carlo and quasi-Monte Carlo methods 2002, pages 27-49. Springer, Berlin, 2004.

[13] E. H. Müller, R. Scheichl, and T. Shardlow. Improving multilevel Monte Carlo for stochastic differential equations with application to the Langevin equation. Proc. $R$. Soc. A, 471, 2015.

[14] E. Novak. Eingeschränkte Monte Carlo-Verfahren zur numerischen Integration. In Mathematical statistics and applications, Vol. B (Bad Tatzmannsdorf, 1983), pages 269-282. Reidel, Dordrecht, 1985.

[15] E. Novak. Deterministic and stochastic error bounds in numerical analysis, volume 1349 of Lecture Notes in Mathematics. Springer-Verlag, Berlin, 1988.

[16] E. Novak. Quantum complexity of integration. J. Complexity, 17(1):2-16, 2001.

[17] E. Novak and H. Pfeiffer. Coin tossing algorithms for integral equations and tractability. Monte Carlo Methods Appl., 10(3-4):491-498, 2004.

[18] S. Omland. Mixed Precision Multilevel Monte Carlo Algorithms for Reconfigurable Hardware Systems. PhD thesis, Technische Universität Kaiserslautern, 2016.

[19] S. Omland, M. Hefter, K. Ritter, C. Brugger, C. De Schryver, N. Wehn, and A. Kostiuk. Exploiting mixed-precision arithmetics in a multilevel Monte Carlo approach on FPGAs. In C. De Schryver, editor, FPGA Based Accelerators for Financial Applications, pages 191-220. Springer Publishing Company, Incorporated, 2015.

[20] J. F. Traub and H. Woźniakowski. The Monte Carlo algorithm with a pseudorandom generator. Math. Comp., 58(197):323-339, 1992.

[21] C. Xu and A. Berger. Best finite constrained approximations of one-dimensional probabilities. ArXiv e-prints, apr 2017.

[22] P. Ye and X. Hu. Optimal integration error on anisotropic classes for restricted Monte Carlo and quantum algorithms. J. Approx. Theory, 150(1):24-47, 2008.

Mathematical Institute, University of Oxford, Oxford OX2 6GG, England

E-mail address: mike.giles@maths.ox.ac.uk

Fachbereich Mathematik, Technische Universität Kaiserslautern, Postfach 3049, 67653 Kaiserslautern, Germany

E-mail address: \{hefter, lmayer,ritter\}@mathematik.uni-kl.de 